\title{
Glycosidic C-O Bond Activation in Cellulose Pyrolysis: Alpha Versus Beta and Condensed Phase Hydroxyl-Catalytic Scission
}

Paul Dauenhauer, Vineet Maliekkal, Matthew Neurock

Submitted date: $12 / 05 / 2020$ - Posted date: 14/05/2020

Licence: CC BY-NC-ND 4.0

Citation information: Dauenhauer, Paul; Maliekkal, Vineet; Neurock, Matthew (2020): Glycosidic C-O Bond Activation in Cellulose Pyrolysis: Alpha Versus Beta and Condensed Phase Hydroxyl-Catalytic Scission. ChemRxiv. Preprint. https://doi.org/10.26434/chemrxiv.12290588.v1

Mechanistic insights into glycosidic bond activation in cellulose pyrolysis were obtained via first principles density functional theory calculations that explain the peculiar similarity in kinetics for different stereochemical glycosidic bonds ( $\beta$ vs $\alpha$ ) and establish the role of the three-dimensional hydroxyl environment around the reaction center in activation dynamics. The reported activating mechanism of the a-isomer was shown to require an initial formation of a transient $\mathrm{C} 1-\mathrm{O} 2-\mathrm{C} 2$ epoxide, that subsequently undergoes transformation to levoglucosan. Density functional theory results from maltose, a model compound for the a-isomer, show that the intramolecular C2 hydroxyl group favorably interacts with lone pair electrons on the ether oxygen atom of an a-glycosidic bond in a manner similar to the hydroxymethyl (C6 hydroxyl) group interacting with the lone pair electrons on the ether oxygen atom of a $\beta$ glycosidic bond. This mechanism has an activation energy of $52.4 \mathrm{kcal} / \mathrm{mol}$, which is similar to the barriers reported for non-catalytic transglycosylation mechanism ( $\sim 50$ $\mathrm{kcal} / \mathrm{mol}$ ). Subsequent constrained ab initio molecular dynamics (AIMD) simulations revealed that vicinal hydroxyl groups in the condensed environment of a reacting carbohydrate melt anchor transition states via two-to-three hydrogen bonds and lead to lower free energy barriers ( 32-37 kcal mol-1) in agreement with previous experiments.

File list (2)

ChemRxiv_Narrative_Vineet_AlphaBeta_ver_05.pdf (0.92 MiB) view on ChemRxiv - download file Vineet_theory_GBactivation_SI_ver_02.pdf (576.67 KiB) view on ChemRxiv - download file 


\title{
Glycosidic C-O Bond Activation in Cellulose Pyrolysis: Alpha Versus Beta and Condensed Phase Hydroxyl-Catalytic Scission
}

\author{
Vineet Maliekkal, Paul J. Dauenhauer, Matthew Neurock*
}

University of Minnesota, Department of Chemical Engineering and Materials Science, 421 Washington Ave. SE, Minneapolis, MN 55455, USA.

*Corresponding author: mneurock@umn.edu

\begin{abstract}
Mechanistic insights into glycosidic bond activation in cellulose pyrolysis were obtained via first principles density functional theory calculations that explain the peculiar similarity in kinetics for different stereochemical glycosidic bonds ( $\beta$ vs $\alpha$ ) and establish the role of the three-dimensional hydroxyl environment around the reaction center in activation dynamics. The reported activating mechanism of the $\alpha$-isomer was shown to require an initial formation of a transient $\mathrm{C}_{1}-\mathrm{O}_{2}-\mathrm{C}_{2}$ epoxide, that subsequently undergoes transformation to levoglucosan. Density functional theory results from maltose, a model compound for the $\alpha$-isomer, show that the intramolecular $\mathrm{C}_{2}$ hydroxyl group favorably interacts with lone pair electrons on the ether oxygen atom of an $\alpha$-glycosidic bond in a manner similar to the hydroxymethyl $\left(\mathrm{C}_{6}\right.$ hydroxyl) group interacting with the lone pair electrons on the ether oxygen atom of a $\beta$ glycosidic bond. This mechanism has an activation energy of $52.4 \mathrm{kcal} / \mathrm{mol}$, which is similar to the barriers reported for non-catalytic transglycosylation mechanism $(\sim 50 \mathrm{kcal} / \mathrm{mol})$. Subsequent constrained ab initio molecular dynamics (AIMD) simulations revealed that vicinal hydroxyl groups in the condensed environment of a reacting carbohydrate melt anchor transition states via two-to-three hydrogen bonds and lead to lower free energy barriers $\left(\sim 32-37 \mathrm{kcal} \mathrm{mol}^{-1}\right)$ in agreement with previous experiments.
\end{abstract}

1.0 Introduction. The thermal deconstruction of cellulose leads to the formation of small oxygenates and sugars that can be upgraded to value-added platform chemicals or to drop-in replacements for conventional fossil fuels. ${ }^{1,2}$ Fast pyrolysis is one such technology used in the deconstruction of cellulose to fuels and chemicals that has gained significant attention. ${ }^{3-6}$ Unlike other bio-based degradation routes, such as enzymatic hydrolysis ${ }^{7,8}$, fast pyrolysis of cellulose can be carried out at significantly higher rates and temperatures, does not involve the use of expensive catalysts or enzymes, and can be integrated with the existing infrastructure in petroleum refineries. ${ }^{9}$ As a result, decades of research efforts have been dedicated towards the realization of fast pyrolysis as a viable commercial technology. The long-standing challenge in this area, however, has been its inability to selectively control specific pyrolysis products. This challenge primarily results from a lack of understanding of the underlying, elementary bond-breaking and bond-making events that comprise pyrolytic chemistry and has been obscured by the structural complexity of the feedstock and the vastness of the complex reaction network. ${ }^{10}$

The cleavage of 1-4 C-O glycosidic bond (i.e., polymeric linkages), which is the first step (atom numbering scheme shown in Figure 1A) in the reaction network is critical in the mechanism of glycosidic bond activation. Accordingly, significant attention has been given to understanding the intra-molecular chemical and electronic interactions that govern this reaction. ${ }^{11}$ Previous theoretical studies have shown that these bonds are primarily activated through concerted reactions $^{12-16}$ rather than heterolytic ${ }^{17,18}$ or homolytic activation mechanisms ${ }^{19}$. Transglycosylation (Figure 1B) is thought to proceed by the concerted formation of an intramolecular $\mathrm{O}^{6}-\mathrm{C}^{1}$ ether bond and cleavage of the $\mathrm{C}^{1}-$ $\mathrm{O}^{1}$ glycosidic bond via an intramolecular proton 
A

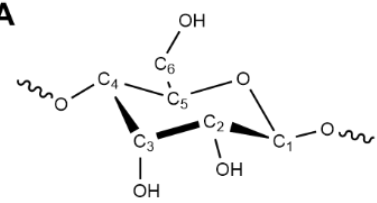

C

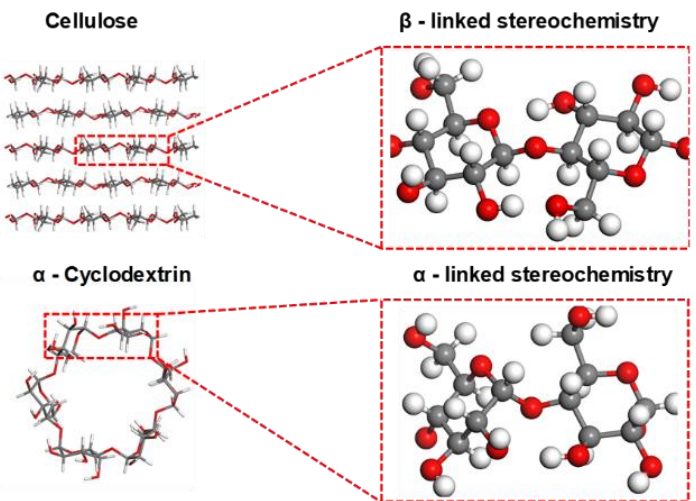

B

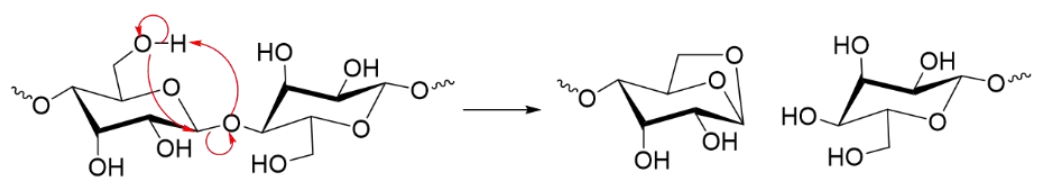

D

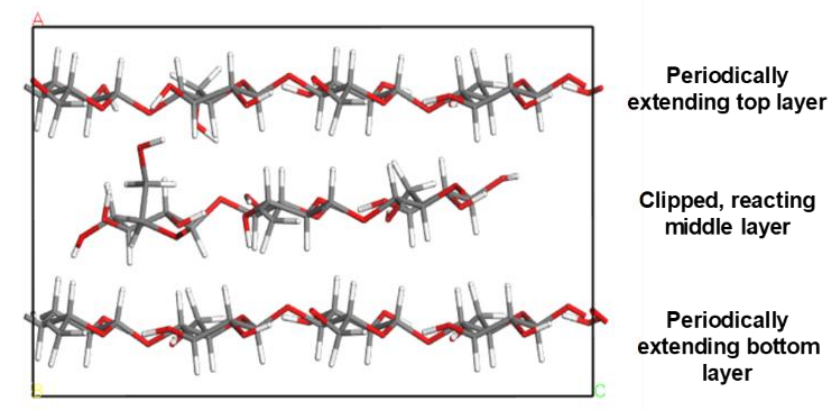

Figure 1. (A) Schematic for numbering of $\mathrm{C}$ atoms, $\mathrm{O}$ and $\mathrm{H}$ atoms are numbered based on the $\mathrm{C}$ atom they are bonded to. (B) Mechanistic scheme for the concerted transglycosylation reaction of $\beta$-stereochemical isomers. (C) Comparative illustration of stereochemistry of $\beta$-linked glycosidic bonds (above equatorial plane) in cellulose versus $\alpha$-linked glycosidic bonds (below equatorial plane) in $\alpha$-cyclodextrin. (D) Illustration of three-layered model system used for all AIMD simulations.

transfer resulting in the formation of a levoglucosan chain end. This is currently considered the dominant mechanism for the activation of cellulose. ${ }^{13-16}$ As a result, deterministic kinetic models have been developed that use the transglycosylation mechanism as the primary mechanism to break down cellulose to anhydrooligomers. ${ }^{11}$ However, as discussed below, the transglycosylation mechanism is still unable to explain certain experimental observations made on initial glycosidic bond activation.

First, experimental studies by Patwardhan et al. have shown that the final chemical product yields are similar for both maltose and disaccharides which have $\alpha$ stereochemical glycosidic bonds, and cellobiose and disaccharides which have $\beta$ stereochemical glycosidic bonds. ${ }^{20}$ In addition, Mettler et al. have shown that cyclodextrin $(\alpha-$ linked polysaccharides) is a kinetic surrogate for cellulose ( $\beta$-linked polysaccharides). ${ }^{21,22}$ However, theoretical calculations performed by Mayes et al. have suggested that inverting the stereochemistry of the glycosidic bonds (Figure 1C), from $\beta$ to $\alpha$, should make it infeasible to carry out the transglycosylation reaction. ${ }^{23}$ To resolve this contradiction, it was proposed that larger polysaccharides would first breakdown into $\alpha$ - glucose via thermohydrolysis. The $\alpha$-glucose could then subsequently undergo a rapid muta-rotation step to change into $\beta$-glucose before undergoing transglycosylation. ${ }^{23}$ Recent work by Maduskar et al. ${ }^{24}$, however, has shown that light molecules, such as water, spontaneously volatilize out of the reactive cellulose melt at operating conditions thus making hydrolysis improbable. This is in line with other studies that have found no evidence for hydrolysis to be a primary pathway. ${ }^{25,26}$ These differences between theory and experiment suggest a need for re-examining the intramolecular bondmaking and bond-breaking events that influence glycosidic bond activation with a special emphasis on explaining the peculiar similarity between $\alpha$ and $\beta$-isomers.

In addition to the influence of the intramolecular structure of fragments, the structure of the local intermolecular reaction environment that forms during reaction dictates the resulting kinetics. The complex arrangements of cellulosic chains can result in the formation of nested hydroxyl environments that can alter the intrinsic reactivity. Modelling studies by Seshadri et al. have shown the potential for hydroxyl groups in water and small alcohols to enhance the conversion of glucose to levoglucosan. ${ }^{27}$ Co-pyrolysis studies 
conducted by Maduskar et al. ${ }^{24}$ also established that hydroxyl-rich additives such as fructose lead to increased levoglucosan yields from cellulose pyrolysis. Furthermore, using thin-film techniques $^{28}$ and the PHASR (Pulse Heated Analysis of Solid Reactions $)^{29}$ microreactor setup, Zhu et al. ${ }^{30}$ provided evidence for a distinct kinetic regime at low temperatures ( 380-470 $\left.{ }^{\circ} \mathrm{C}\right)$ characterized by energetics that are atypical of thermal mechanisms such as transglycosylation. More recently, we used isotopic labeling and experimental PHASR studies along with density functional theory (DFT) calculations to show that the primary mechanism for glycosidic bond activation at these low temperatures proceeds through site specific, vicinal hydroxyl catalyzed transglycosylation. ${ }^{31}$ These studies have collectively demonstrated that the specific local hydroxyl environments present within the cellulose matrix can promote its reactivity and open up new pathways that are different from previously considered thermal pathways. Understanding the influence of these environments on the measured reactivity, however, is challenging due to the dynamics of the local cellulose structure as noted in a recent review. ${ }^{32}$ The previous DFT studies discussed above have used static clusters to examine the effects of vicinal hydroxyl groups, and therefore little is known about the dynamic changes that occur at high temperatures, the entropies and free energies associated with such changes, or their influence on the reaction kinetics.

As discussed above, resolving these gaps in our understanding of the mechanism for glycosidic activation requires deeper insights into the structural and chemical factors, intra-molecular bond making-breaking events at the reaction center, as well as intermolecular condensed-phase environment interactions that affect the energetics of glycosidic bond activation. In this study, we carry out first principles DFT calculations to investigate thermal, gas-phase pathways, i.e. reaction paths that initially ignore the effects of the surrounding environment in the depolymerization of cellulose. This includes understanding the electronic interactions that govern bond breaking and bond making in the transglycosylation pathway and then developing a mechanism for $\alpha$-linked species that displays similar facile, electronic interactions. Such an approach presents a detailed molecular understanding of thermal depolymerization pathways that are expected to dominate at higher operating temperatures. We subsequently examine the role of the local reaction environment and the influence of intermolecular interactions via modeling of condensed phase glycosidic activation and follow up on our earlier studies $^{31}$. More specifically, constrained ab initio molecular dynamics (AIMD) simulations are used to capture the dynamics of local reaction environment and re-organization of vicinal hydroxyl groups present in the melt phase to catalyze depolymerization. Our simulations show that these catalyzed pathways proceed only at specific sites and that the activity of these different sites varies based on the nature of the environment. Thus, through the use of AIMD simulations we help to elucidate important differences between thermal, gas-phase pathways and condensed-phase, hydroxyl catalytic pathways. More broadly, the theoretical insights presented herein taken along with the existing mechanistic knowledge available in the literature provides a more detailed understanding of glycosidic activation and presents a framework for investigation of other reactions that are involved in the pyrolytic reaction network of cellulose.

2.0 Computational Methods. All of the simulation results reported herein were carried out using periodic plane-wave density functional theory (DFT) as implemented in Vienna $\mathrm{Ab}$ initio Simulation Program (VASP). ${ }^{33-35}$ The generalized gradient approximation (GGA) form of the Perdew, Burke and Ernzerhof $(\mathrm{PBE})^{36}$ functional was used to calculate exchange correlation energies along with D2 corrections developed by Grimme ${ }^{37,38}$ to account for dispersion corrections. Plane waves were constructed using an energy cutoff of $396 \mathrm{eV}$ along with projector augmented wave potentials (PAW) to model interactions between core and valence electrons. ${ }^{39,40}$ The core model system used to elucidate the activation mechanism in $\alpha$ linked species consists of a maltose molecule in a large $18 \times 18 \times 18 \AA$ unit cell to resemble a gas phase environment. A $1 \times 1 \times 1 \mathrm{k}$-points grid ${ }^{41}$ was chosen based on energy convergence tests and used for all calculations. The calculations were carried out to converge the self-consistent field and geometries to within $10^{-6} \mathrm{eV}$ and forces to within $0.02 \mathrm{eV} / \AA$. The climbing NEB method ${ }^{42,43}$ was used to generate the intermediate images along the reaction coordinate 
and subsequently optimize the reaction path with a force tolerance of $0.2 \mathrm{eV} / \AA$. The transition state was subsequently isolated by using the highest energy images and the dimer method with a force tolerance of $0.02 \mathrm{eV} / \AA^{44}$

Frequency calculations were performed on the maltose system to determine the free energies for the $\alpha$ glycosidic bond activation mechanism. These frequencies were subsequently used to calculate zero-point energy corrections as well as enthalpic and entropic contributions from vibrations as per the quasi-harmonic oscillations approach. Low value frequencies and spurious imaginary frequencies that have been reported in periodic systems were treated using a cut-off frequency whereby all such vibration modes are assumed to have a vibrational frequency of $100 \mathrm{~cm}^{-1}$ as has been done in previous studies in the literature. ${ }^{45}$

The AIMD simulations reported herein were also carried out using the Vienna $\mathrm{Ab}$ initio Simulation Program. The initial structures for the system were constructed using the crystal structure of cellulose I $\beta .^{46,47}$ The model system involved 3 distinct layers as shown in Figure 1D above, with the middle layer being the layer undergoing activation. The top and bottom layer extend periodically along the $\mathrm{c}$ axis, with 4 monomers lying inside the simulation cell for each of these two layers, to represent infinitely long chains. The middle, reacting layer, however, was clipped at the ends to give an effective chain length of 3 monomers inside the simulation cell. This clipping was necessary as a periodically extending chain cannot be cleaved due to symmetry restrictions. It also leads to the creation of chain-end and midchain positions thus allowing us to explore reactivity at these two positions. To replicate interchain H-bonding arrangements as in the crystal and to attain an appropriate density, the 3 layers were placed in a monoclinic periodic simulation cell with $r=96.55^{\circ}$, in close agreement with experiments $^{46,47}$, and lattice parameters $\mathrm{a}=12.568$ $\AA, \mathrm{b}=8.201 \AA$ and $\mathrm{c}=20.760 \AA$.

The AIMD simulations were carried out using an NVT ensemble with temperature being maintained at $670 \mathrm{~K}$ and a Nose'-Hoover thermostat. ${ }^{48,49}$ This temperature was chosen as it lies in the middle of the experimental temperature range observed for the catalyzed activation regime as reported by Zhu et al. ${ }^{30}$ To reduce computational cost, the self-consistent field calculations were carried out to converge the energies to within $10^{-4}$ eV. A 1 fs time step was used for the AIMD simulations. It has been reported that 1 fs time step may be too large and could lead to thermal separation during $\mathrm{C}-\mathrm{H}$ or $\mathrm{O}-\mathrm{H}$ stretching. ${ }^{50}$ To avoid such separations while using a large time step, the mass of $\mathrm{H}$ atoms is taken to be equal to that of tritium as is done in previously reported studies. ${ }^{50}$ Constrained AIMD simulations were carried out using the SHAKE algorithm. ${ }^{51}$ The accuracy of constrained AIMD calculations depends not only on the choice of constrained reaction coordinates but also on the values of the chosen constraints. An over constrained system can lead to inaccurate free energies whereas a poor choice of the values for the constraints can lead to errors in the simulation. Therefore, a systematic approach was used to guide our choice of constraints and their values. The climbing NEB method was used to obtain an optimized trajectory to establish the progress of reaction. The reaction constrained coordinates were then chosen and the resulting intermediate images from the NEB were used to obtain values of the constraint.

Bias introduced into the system due to imposed constraints was corrected using the Blue moon sampling approach to provide statistically accurate mean free energy gradients and constraint forces $\left\langle\mathrm{F}_{\mathrm{c}}\right\rangle{ }^{52-54}$ The system is first equilibrated for $5 \mathrm{ps}$ and then $10 \mathrm{ps}$ of additional simulation is performed to sample constraint forces. Longer simulations up to 22 ps were carried out for the chain end reactions and no changes were observed in the mean constraint forces thus indicating that 15 ps provided reliable constraint forces. The $\left\langle\mathrm{F}_{\mathrm{c}}\right\rangle$ - reaction coordinate plot for these longer simulations are reported in the SI. Helmholtz free energies were calculated from the obtained $\left\langle\mathrm{F}_{\mathrm{c}}\right\rangle$ by performing thermodynamic integration ${ }^{55}$ using the trapezoidal rule. Since we are dealing with a condensed phase system, Helmholtz free energies are not expected to be significantly different from Gibbs free energies.

3.0 Results and Discussion. To better understand the bond-breaking and bond-making events involved in cellulose depolymerization and explain the experimental observations discussed in the introduction, we examined the influence of the intra-molecular interactions as well as the role of the condensed-phase environment on these reactions. More specifically, a detailed analysis of 

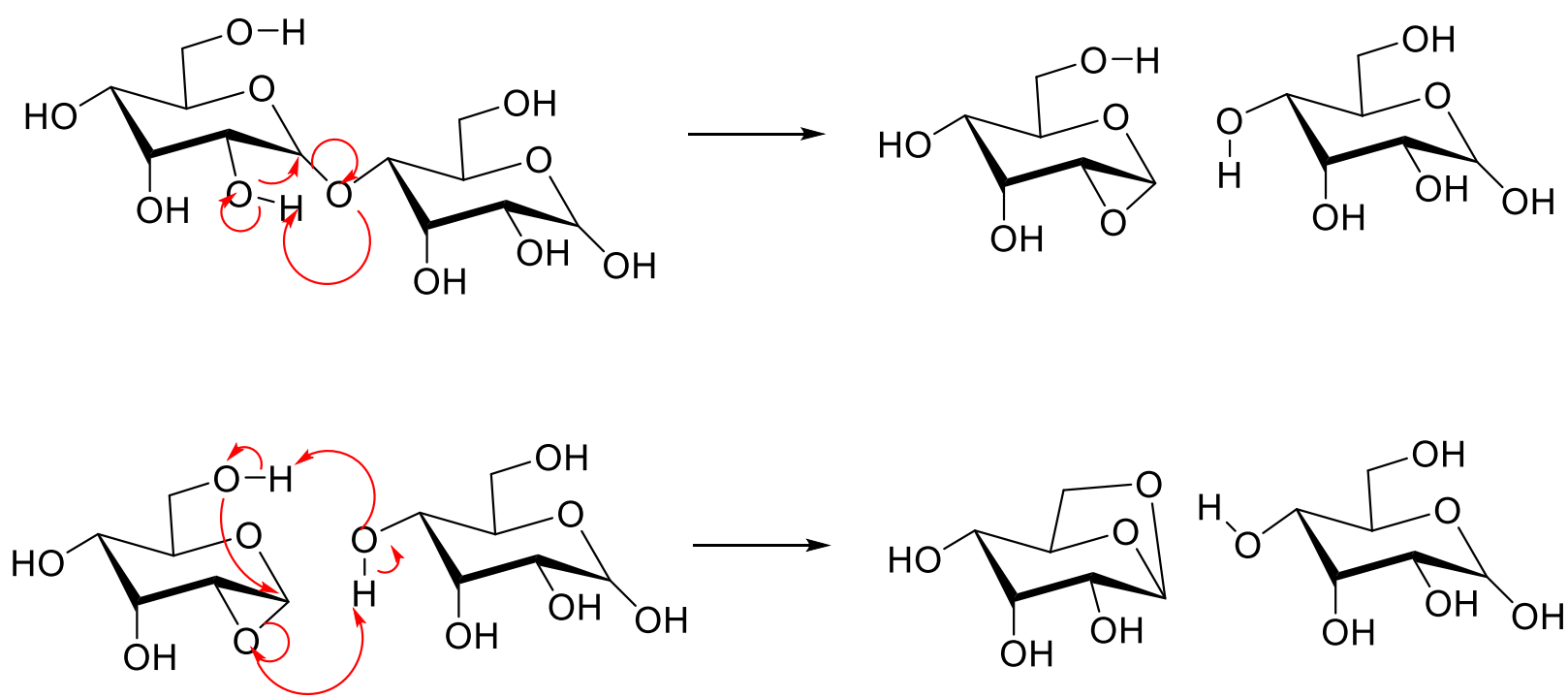

Scheme 1. Proposed mechanistic scheme for activating $\alpha$-glycosidic bonds in model compound maltose proceeds via attack by the $\mathrm{C}_{2}$ hydroxyl group.

the transglycosylation mechanism to cleave $\beta$ glycosidic bonds is presented below in section 3.1. The insights from these results indicate that the activation of the $\alpha$-glycosidic bond proceeds via a mechanism that is similar to that in activating the $\beta$ glycosidic bond. The activation of the $\alpha$-glycosidic bond proceeds via an intra-molecular addition of the $\mathrm{O}_{2}$-hydroxyl to $\mathrm{C}_{1}$ glycosidic $\mathrm{C}-\mathrm{O}$ bond. We examine the free energies for this thermal depolymerization mechanism and separate enthalpic and entropic effects. In section 3.2, we examine the influence of the condensed phase in cellulose depolymerization via constrained AIMD simulations. The AIMD simulations carry out thermodynamic integration along a constrained reaction coordinate to determine the free energy barriers and the influence of vicinal $\mathrm{OH}$ groups in catalyzing the transglycosylation of the $\beta$ glycosidic bonds. Lastly, the AIMD simulations are extended in section $\mathbf{3 . 3}$ to explore reactivity of different regions of cellulose - active cellulose sites, non-reducing chain end sites, mid-chain internal sites (levoglucosan chain ends).

\subsection{Intramolecular interactions influencing} $\beta$ vs $\alpha$ glycosidic activation chemistry. We initially examine the efficacy of transglycosylation which has been proposed previously as a mechanism for glycosidic bond activation. As was discussed in section 1.0, the transglycosylation of $\beta$ glycosidic bonds can readily proceed via the concerted addition of the $\mathrm{O}^{6}$ oxygen of the hydroxymethyl to the $\mathrm{C}^{1}$ ether linkage, cleavage of the $\mathrm{C}^{1}-\mathrm{O}^{1}$ bond, and proton transfer from the $\mathrm{O}^{6}-\mathrm{H}$ hydroxyl to the $\mathrm{O}^{1}$ ether linkage to form the levoglucosan chain end. The $\alpha$ glycosidic linkage, however, has an inverted structure at the $\mathrm{C}^{1}$ site which places the $\mathrm{O}^{1}$ oxygen below the glycosidic plane thus preventing proton transfer and the direct addition of $\mathrm{O}^{6} \mathrm{H}$ to the $\mathrm{C}^{1}$. While ionic and radical reactions are possible, the barriers for these paths, as was discussed in section 1.0, are considerably higher than those for the concerted mechanism which rule these reactions out.

A closer analysis of the $\beta$ glycosidic bond structure in Figure $\mathbf{B B}$ indicates that the concerted mechanism can proceed but requires a proton source close to the $\mathrm{O}^{1}$ linkage. This source can either be from a vicinal $\mathrm{O}-\mathrm{H}$ bond (as in transglycosylation) or a $\mathrm{C}-\mathrm{H}$ bond (as in glycosylation ${ }^{12}$ ). The $\mathrm{C}-\mathrm{H}$ hydrogen, however, is much less acidic than of that of the $\mathrm{O}-\mathrm{H}$ hydrogen and is likely why the glycosylation path is less likely than transglycosylation. A closer examination of the transglycosylation mechanism indicates that the reaction involves the interaction of the proton from the $\mathrm{C}_{6}$ hydroxymethyl $\mathrm{O}-\mathrm{H}$ group with the lone pair electrons of the glycosidic oxygen atom along with the simultaneous nucleophilic attack of the hydroxymethyl $\mathrm{O}_{6}$ on the cationic $\mathrm{C}_{1}$ atom. The strength of interaction between the hydroxymethyl $\mathrm{O}_{6}-\mathrm{H}$ proton and the lone pair of electrons on the $\mathrm{O}_{1}$ together with the 

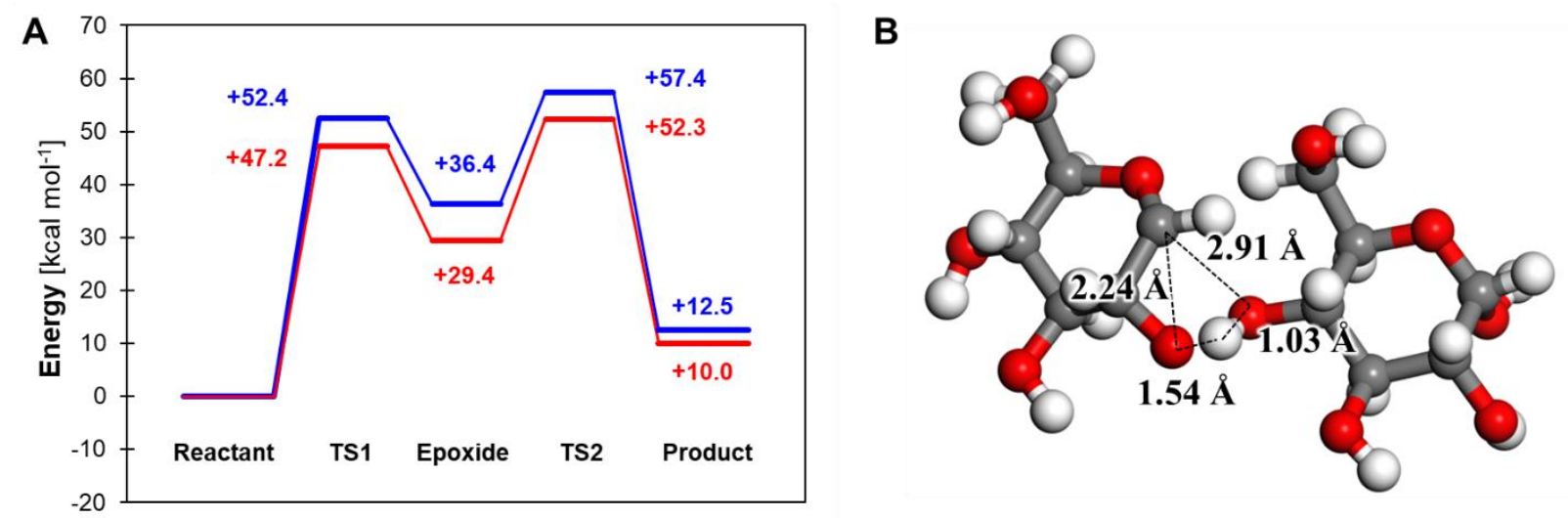

Figure 2. (A) Calculated electronic (blue) and free energy (red) profiles for the mechanism proposed in scheme 1 with activation energy of $52.4 \mathrm{kcal} / \mathrm{mol}$ for initial glycosidic bond activation in maltose, similar to $\beta$-linked species. (B) Optimized transition state for the first step of glycosidic activation.

ease of the nucleophilic addition of the $\mathrm{O}_{6}$ to $\mathrm{C}_{1}$ strongly influence the activation barrier and hence the kinetics of activation.

In the case of $\beta$ linkages, the lone pair on the glycosidic $\mathrm{O}$ is oriented above the equatorial plane thus making the interaction of the hydroxymethyl group (also oriented above the plane) much easier than any other intra-molecular hydroxyl group. In the case of $\alpha$-linked species, even though a similar nucleophilic attack seems feasible, the lone pair of electrons on the glycosidic oxygen point below the equatorial plane which prevents their interaction with the proton of the hydroxymethyl group. In the absence of this interaction, C-O activation via transglycosylation cannot occur. A reasonable mechanism for activating $\alpha$ glycosidic bonds therefore will involve not just stabilization via nucleophilic attack but also favorable interaction with lone pair electrons on the glycosidic oxygen.

The importance of a sterically favorable interaction between the attacking proton and glycosidic oxygen lone pair electrons motivated a closer look at other intra-molecular hydroxyl groups that were better positioned to interact with lone pair electrons and activate the $\alpha$ glycosidic C$\mathrm{O}$ bond. The $\mathrm{C}_{2}$ hydroxyl group, which is oriented below the equatorial plane, can favorably interact with glycosidic $\mathrm{O}$ as well as carry out a nucleophilic attack on the $\mathrm{C}_{1}$ atom. A mechanism for levoglucosan formation via glycosidic bond activation can therefore be envisioned that proceeds in two steps (Scheme 1) as follows,

(1) Step 1 - Glycosidic bond cleavage activated by $\mathrm{C}_{2}$ hydroxyl group resulting in the formation of an epoxide intermediate. This step involves the concerted addition of the $\mathrm{O}_{2}$ hydroxyl to the adjacent $\mathrm{C}_{1}$ site, $\mathrm{C}_{1}-\mathrm{O}_{1}$ activation, and proton transfer from $\mathrm{O}_{2}$ to $\mathrm{O}_{1}$. A similar mechanism for the $\beta$-linked species that involved an $\mathrm{S}_{\mathrm{N}} 2$ attack by $\mathrm{C}_{2}$ hydroxyl group on $\mathrm{C}_{1}$ was proposed previously, but this mechanism was shown to be unfavorable as the migration path of the proton from the $\mathrm{C}_{2}$ hydroxyl group to glycosidic $\mathrm{O}$ in the $\beta$-linked species is highly strained. In contrast, the glycosidic $\mathrm{O}$ and $\mathrm{C}_{2}$ hydroxyl group in the $\alpha$-linked species are oriented in the same direction thus leading to facile proton transfer.

(2) Step 2 - Formation of levoglucosan from the epoxide intermediate via proton transfer from $\mathrm{C}_{6}$ hydroxymethyl group to the epoxide $\mathrm{O}$ via free $\mathrm{C}_{4}-\mathrm{OH}$ of detached glucose. The assistance of the terminal hydroxyl group from the detached glucose unit in the second step to form levoglucosan was found to be essential; in the absence of this assistance, the calculated activation barrier was significantly higher $(82.4$ $\mathrm{kcal} / \mathrm{mol}$, transition state structure available in SI Figure S-2) than that expected from experiments. This high barrier is the result of significant strain in directly transferring the hydroxymethyl proton to the epoxide oxygen. The assistance from the $\mathrm{C}_{4}-\mathrm{OH}$ group of the detached glucose aids the levoglucosan formation by enabling facile proton shuttling to the epoxide thus lowering the barrier.

This mechanism was validated by using maltose as a model compound as it is the smallest 
sugar molecule with an $\alpha$-linked stereochemistry for the glycosidic oxygen. DFT calculations (Figure 2A) reveal an activation barrier of 52.4 $\mathrm{kcal} / \mathrm{mol}$ for the first step which involves the formation of the glycosidic activation and epoxide formation. The calculated barrier agrees well with experimental barriers measured by Zhu et al. ${ }^{30}$ for cyclodextrin breakdown kinetics at temperatures higher than $467{ }^{\circ} \mathrm{C}(53.7 \pm 1.1 \mathrm{kcal} / \mathrm{mol})$. This barrier also agrees reasonably well with the calculated barriers for transglycosylation of cellobiose (46-53 kcal/mol). ${ }^{13-15}$ In addition, it also agrees with barriers for transglycosylation reported in one of our recent studies using functionals similar to those in this study. The subsequent reaction step involving the conversion of the epoxide intermediate to levoglucosan has an activation energy of $21.0 \mathrm{kcal} / \mathrm{mol}$.

A close look at the transition state for the first step of C-O activation in maltose (Figure 2B) reveals similarities with the transition state for the concerted transglycosylation for $\beta$-linked species. The $\mathrm{O}_{2}-\mathrm{H}_{2}$ and $\mathrm{H}_{2}-\mathrm{O}_{\text {glycosidic }}$ bond distances are 1.54 and $1.03 \AA$, respectively, in maltose $\mathrm{C}-\mathrm{O}$ activation which compares well with the $\mathrm{O}_{6}-\mathrm{H}_{6}$ and $\mathrm{H}_{6^{-}}$ $\mathrm{O}_{\text {glycosidic }}$ bond distances of 1.50 and $1.03 \AA$, respectively, reported in our initial study of noncatalytic transglycosylation in cellobiose. ${ }^{31}$ The cationic $\mathrm{C}_{1}$ center that forms in the transition state for maltose examined herein (Figure 2B) is stabilized by the anionic $\mathrm{O}_{2}$ and the cleaved glycosidic O1. This is similar to the transition state for the transglycosylation, where the cationic $\mathrm{C}_{1}$ is stabilized by the anionic $\mathrm{O}_{6}$ and the cleaved glycosidic $\mathrm{O}_{1}$. These findings suggest that the two intramolecular hydroxyl groups, $\mathrm{C}_{6}$ hydroxymethyl and $\mathrm{C}_{2}$ hydroxyl, are capable of carrying out similar roles, namely favorable interactions with lone pairs and stabilization via nucleophilic attack on $\mathrm{C}_{1}$ cationic center, with regards to cleaving $\beta$ versus $\alpha$ glycosidic bonds, respectively, and would lead to comparable kinetics when cleaving glycosidic bonds with different stereochemistry.

In addition to the electronic energies reported above, the free energies for the different intermediates and transition states in the mechanism above were calculated using the vibration frequencies via the quasi-harmonic oscillator approach. As depicted in Figure $\mathbf{2 A}$, the free energy of activation for the first step is 47.2 $\mathrm{kcal} / \mathrm{mol}$ which indicates that there is an entropic gain of $6.7 \mathrm{cal} / \mathrm{mol}-\mathrm{K}$. Such an entropic gain is expected as there is an increase in the degrees of freedom as the disaccharide is cracked to smaller monosaccharides at high temperatures. The transition state in Figure $2 \mathrm{~B}$ also shows that the $\mathrm{C}_{1^{-}}$ $\mathrm{O}_{1}$ glycosidic bond is fully cleaved (bond distance $=2.91 \AA$ ) further justifying the observed entropic gains. The second step shows a free energy of activation of $22.9 \mathrm{kcal} / \mathrm{mol}$ at $500{ }^{\circ} \mathrm{C}$ while the formation of the epoxide intermediate has an endothermic free energy of reaction of 29.4 $\mathrm{kcal} / \mathrm{mol}$. The highly endothermic nature of the first step implies that the epoxide intermediate is not stable and is likely to undergo immediate transformation to other products such as levoglucosan. Experimentally isolating this epoxide intermediate would be challenging, consistent with no reported experimental observations of this species..$^{21,22}$

The above results establish a mechanism that proceeds with similar activation energies as the transglycosylation mechanism and can thus explain the similarities found in the activation of molecular structures with stereo-chemically unique $\alpha$ - and $\beta$ glycosidic bonds and their depolymerization kinetics. The calculated free energies suggest significant gains in entropy as the reactions are carried out in the gas phase. The extent of these entropic gains in the melt phase with a confined environment will likely be less significant. In order to examine the influence of the melt phase and the dynamics of the reaction environment, simulations were further conducted via constrained ab initio molecular dynamics (AIMD).

3.2 Free energy barriers for hydroxylcatalyzed transglycosylation in melt phase. The free energies and entropies of activation for glycosidic bond activation in the melt phase environment were calculated using constrained AIMD simulations and larger periodic models of the local reaction environment in the melt phase. In particular, the hydroxyl-catalyzed mechanism introduced earlier ${ }^{31}$ for $\beta$-glycosidic linkages was considered to elucidate the roles that vicinal hydroxyl groups can play by directly participating in bond breaking and making events. This mechanism consists of two steps: (1) a quasiequilibrated binding step which involves the formation of hydrogen bonds between the reaction center and the vicinal hydroxyl groups, and subsequently (2) a slower rate-determining step 


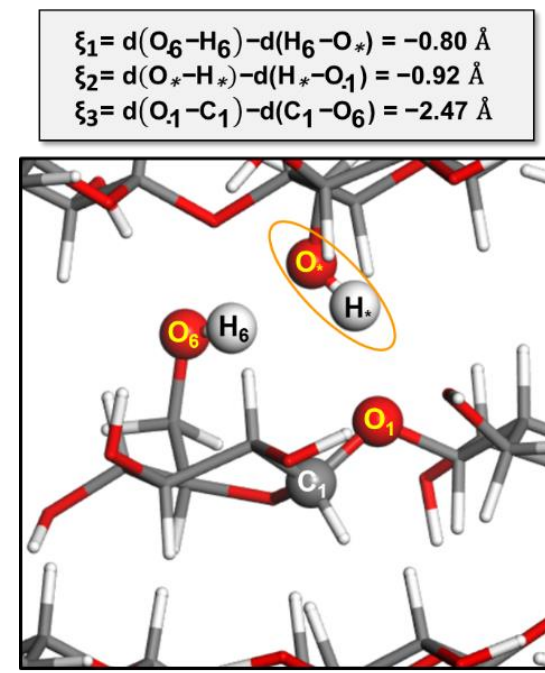

(A) Bound reactant

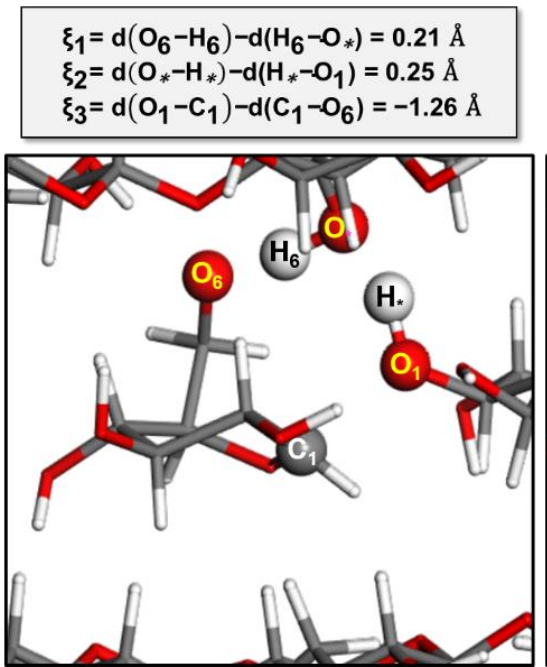

(B) Intermediate image

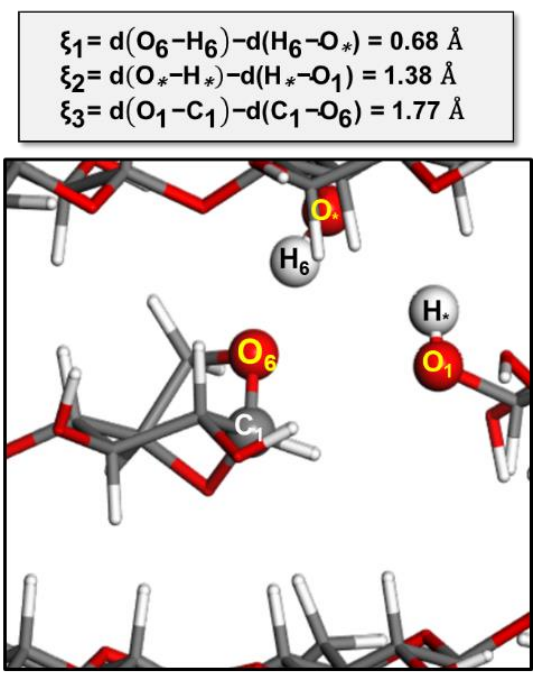

(C) Bound product

Figure 3. Changes in participating bonds along the reaction coordinate captured by three selected constraints. Evolution of constraint values shown for (A) bound reactant, $(\mathbf{B})$ intermediate image, and $(\mathbf{C})$ bound product along the reaction path. Labeled atoms constitute atoms that make up the reaction center.

which carries out transglycosylation while explicitly using one of the bound hydroxyl groups as a proton shuttling bridge.

The DFT-optimized starting configuration for constrained AIMD simulations to determine the free energy barrier is shown in Figure 3A. In this initial state, the $\mathrm{C}_{6}$ hydroxymethyl group on the non-reducing chain end is hydrogen bonded to one of the vicinal hydroxyl groups $(\mathrm{O} * \mathrm{H} *$ circled in orange in the bound reactant in Figure 3A), which in turn is hydrogen bonded to the glycosidic ether oxygen $\mathrm{O}_{1}$ to form a proton shuttling bridge required for reaction. The initial reactant and product states (Figure $3 \boldsymbol{A}$ and $3 \boldsymbol{C}$ ) for the glycosidic bond activation were optimized to provide the lowest energy reactant and product states. A climbing nudged elastic band (NEB) simulation was subsequently carried out using these optimized states along with 18 intermediate images to determine the reaction coordinate. The optimized trajectory of the NEB revealed the nature of the multi-dimensional reaction coordinate. The mechanism consisted of three events: (i) proton transfer between hydroxymethyl $\mathrm{O}_{6}-\mathrm{H}_{6}$ to the vicinal $\mathrm{O} *-\mathrm{H}_{*}$, (ii) proton transfer between the vicinal $\mathrm{O} *-\mathrm{H}_{*}$ and glycosidic $\mathrm{O}_{1}$, and (iii) cleaving $\mathrm{C}_{1}-\mathrm{O}_{1}$ and forming $\mathrm{C}_{1}-\mathrm{O}_{6}$ bond. These three events occurred simultaneously in a concerted fashion. This led to the following choice for three selected constrained reaction coordinates,

$$
\begin{aligned}
& \xi_{1}=\mathrm{d}\left(\mathrm{O}_{6}-\mathrm{H}_{6}\right)-\mathrm{d}\left(\mathrm{H}_{6}-\mathrm{O}_{*}\right) \\
& \xi_{2}=\mathrm{d}\left(\mathrm{O}_{*}-\mathrm{H}_{*}\right)-\mathrm{d}\left(\mathrm{H}_{*}-\mathrm{O}_{1}\right) \\
& \xi_{3}=\mathrm{d}\left(\mathrm{O}_{1}-\mathrm{C}_{1}\right)-\mathrm{d}\left(\mathrm{C}_{1}-\mathrm{O}_{6}\right)
\end{aligned}
$$

where $d(x-y)$ refers to bond length between atoms $x$ and $\mathrm{y}$ and $\xi_{1}, \xi_{2}$ and $\xi_{3}$ refer to the constrained reaction coordinates. Figures $\mathbf{3 A}, \mathbf{B B}$ and $\mathbf{3 C}$ show the progress of the reaction from the bound reactant state to intermediate image to bound product state, thus describing the above-mentioned reaction coordinates as well as the change in the values of these three chosen constraints as the reaction proceeds. The lower and upper bounds on these three constraints, as described in the bound reactant and product state, are $-0.80 \& 0.68 \AA$, $-0.92 \& 1.38$ $\AA$, and $-2.47 \& 1.77 \AA$, respectively. To decrease computational costs, only 10 out of the 18 intermediate NEB images were selected to obtain initial structures and constraint values for sample points along the reaction coordinate to carry out constrained AIMD simulations.

Figures $4 A, 4 B$ and $4 C$ display the mean constraint force $\left\langle\mathrm{F}_{\mathrm{C}}\right\rangle$ plotted versus the values of the constraints $\xi_{1}, \xi_{2}$, and $\xi_{3}$, respectively. All three plots show that the constraint force on the first image is low and close to zero, indicating that the reacting monomer, which is in a chair conformation bound to the bridging $\mathrm{O} * \mathrm{H} *$ hydroxyl, is a stable minimum structure. This validates the starting configuration as a reasonable initial structure. 

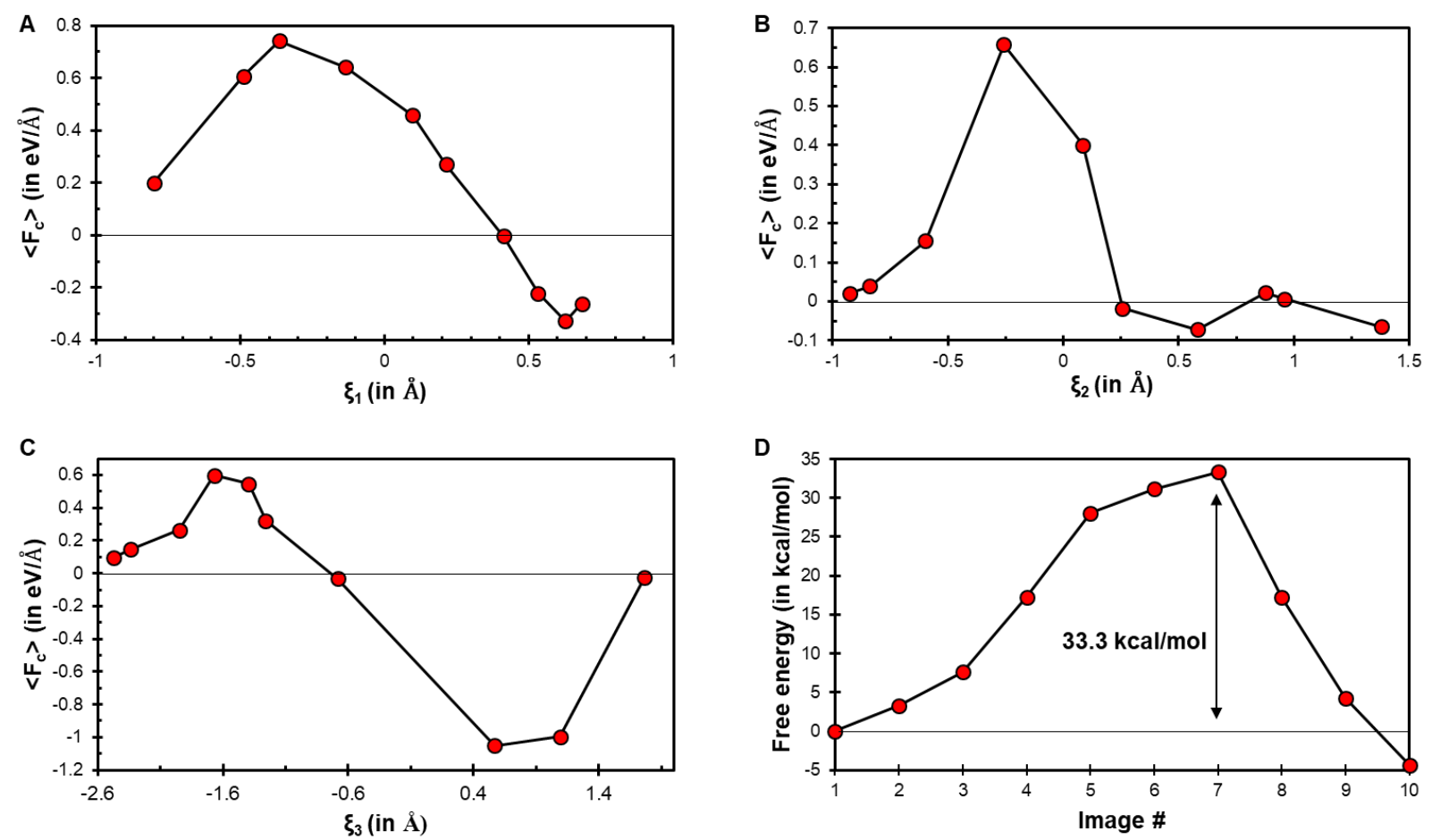

Figure 4. Comparison for mean constraint forces $\langle\mathrm{Fc}\rangle$ versus (A) $\xi_{1}$, (B) $\xi_{2}$, and (C) $\xi 3$ calculated from constrained AIMD simulations. (D) Free energy profile obtained from thermodynamic integration of mean constraint forces reveals a free energy barrier of $33.3 \mathrm{kcal} / \mathrm{mol}$.

Secondly, the shape of the curves displays a characteristic behavior changing from positive to negative forces; the point of cross-over from positive to negative being the transition state. Based on this observation, intermediate image \#7 of Figure $4 D$ appears to be close to the transition state with the $\left\langle\mathrm{F}_{\mathrm{C}}\right\rangle$ being $-0.003,-0.072$ and -0.031 $\mathrm{eV} / \AA$, respectively, along the three constrained coordinates. In this possible transition state structure, the values of the constraints are 0.41 , 0.58 , and $-0.68 \AA$, respectively, which suggests that proton transfer is nearly complete and that the bond between $\mathrm{O}_{6}$ and $\mathrm{C}_{1}$ has not formed while the glycosidic bond is broken. This results in an anionic $\mathrm{O}_{6}$ and cationic $\mathrm{C}_{1}$ which is in qualitative agreement with transition state obtained by static DFT calculations in our previous study. ${ }^{31}$ The values of the same constrained variables in the transition state obtained from the above-mentioned DFT cluster model are $0.57,0.75$, and $-0.47 \AA$ suggesting that high temperature, condensed-phase AIMD predicts a slightly earlier transition state than that from the static DFT calculations. The thermodynamic integration of the above $\left\langle\mathrm{F}_{\mathrm{c}}\right\rangle-\xi$ plots results in an intrinsic free energy of activation of $33.3 \mathrm{kcal} / \mathrm{mol}$
(Figure 4D). These free energy barriers are significantly lower than the reported barriers for thermal activation of glycosidic bonds via noncatalyzed transglycosylation $(\sim 53 \mathrm{kcal} / \mathrm{mol}) .{ }^{13}$ This indicates that at low temperatures the presence of local hydroxyl groups enables the catalytic activation of glycosidic bonds over thermal activation. The $33.3 \mathrm{kcal} / \mathrm{mol}$ free energy barrier reported here is similar to the $32.1 \mathrm{kcal} / \mathrm{mol}$ enthalpic barrier reported previously ${ }^{31}$, thus suggesting that there is a small entropic penalty of $1.64 \mathrm{cal} / \mathrm{mol}-\mathrm{K}$ in activating the bound reactant state.

To understand the observed low free energy of activation as well as the observed entropic penalty, we examined in detail the evolution trajectory of the transition state during the AIMD simulation. The results in Figure 5, which show snapshots of the transition state along the trajectory at $\mathrm{t}=7.5 \mathrm{ps}$ and $15 \mathrm{ps}$, reveal the formation of the anionic $\mathrm{O}_{6}$ and cationic $\mathrm{C}_{1}$ centers in the transition state. Of interest is the interaction of the anionic $\mathrm{O}_{6}$ center with the hydroxyl species present in its surrounding environment. Firstly, a strong H-bonding interaction exists with the bridging $\mathrm{O} *-\mathrm{H} *$ hydroxyl. 

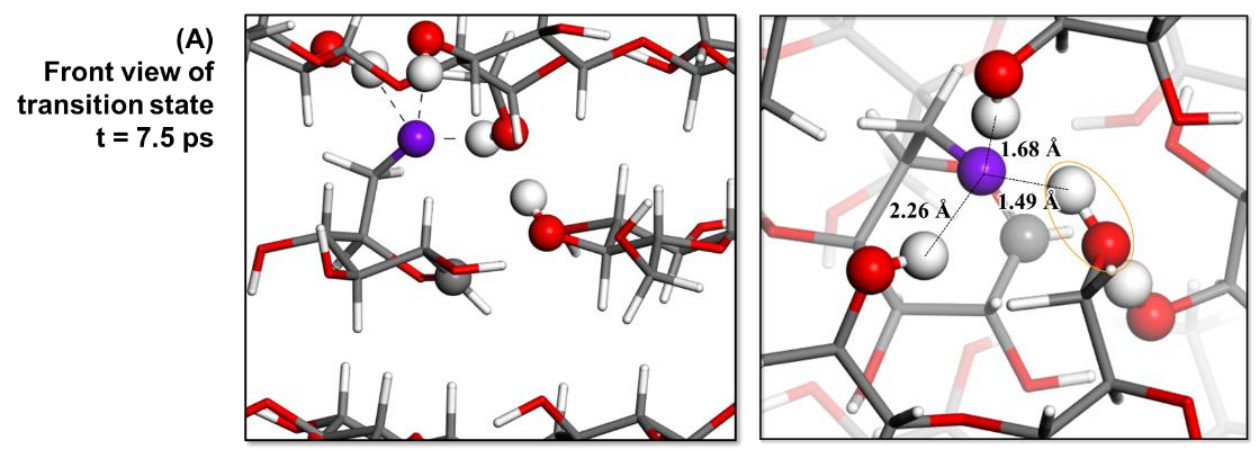

(B) Zoomed in top view of transition state $\mathrm{t}=7.5 \mathrm{ps}$

(C)

Front view of transition state $\mathrm{t}=\mathbf{1 5} \mathrm{ps}$
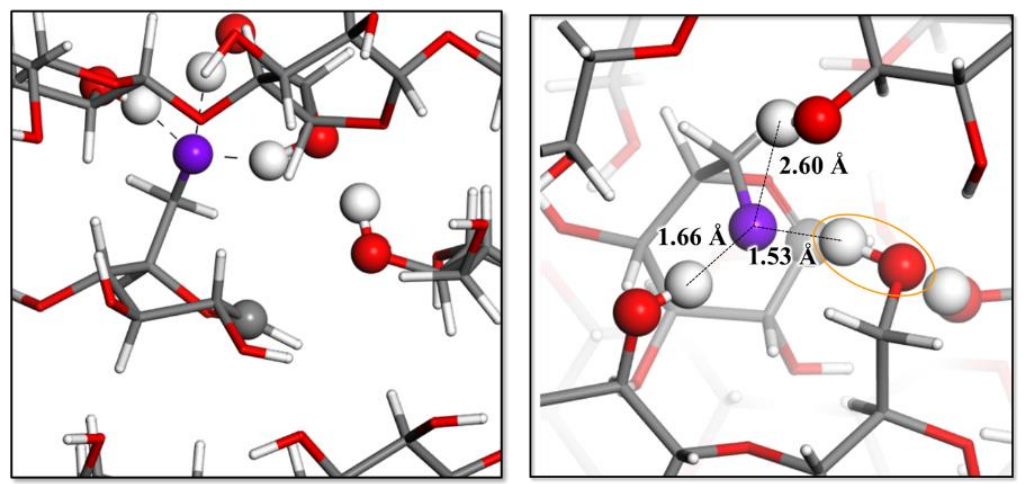

(D) Zoomed in top view of transition state $t=15$ ps

Figure 5. Front view and magnified top view of snapshots of the TS for hydroxyl catalyzed transglycosylation at $\mathrm{t}=$ 7.5 and 15 ps show the presence of three $\mathrm{OH}$ groups around charged centers. Atoms critical to reaction are shown in ball and stick form. The anionic $\mathrm{O}_{6}$ is shown as a purple sphere. Bridging hydroxyl is circled red to differentiate from other two vicinal hydroxyl that order around anionic $\mathrm{O}_{6}$.

Such a strong interaction is expected as the hydroxyl group was constrained to act as proton shuttling bridge. However, in addition to this group, two additional hydroxyl groups also show weak $\mathrm{H}-$ bonding interactions with the anionic $\mathrm{O}_{6}$ center. Unlike the bridging hydroxyl, these two hydroxyl groups are not constrained to interact with the reaction center. This indicates that vicinal hydroxyl groups dynamically organize to form nested pockets/sites to which charged transition states can anchor. This anchoring helps to stabilize the transition state through unique hydrogen bonding networks and electrostatic interactions.

This ordering of hydroxyl groups around active centers to stabilize transition states appears to be qualitatively similar to unique ordering of solvents around active centers that has been observed in recent studies on acid-catalyzed dehydration reactions in solvent mixtures. ${ }^{56,57}$ These AIMD results suggest that even in a solid/molten cellulosic matrix, which is not physically similar to a conventional homogeneous solvent system, local hydroxyl groups tend to arrange around charged $\mathrm{O}$ centers as nested sites to attain the increased stability offered by such H-bonding networks.
While these networks lead to significant electronic stabilization of the transition state over the reactant, the ordered nature of these $\mathrm{H}$-bonds and the strong binding of the transition state relative to that of the reactant state would decrease the degrees of freedom and would help to explain the small entropic penalties observed in the simulations. More broadly, the results of this section show the critical importance of explicit inclusion of the condensed phase in capturing kinetics and chemistry.

3.3 Free energy trends for hydroxylcatalyzed reaction at different regions of cellulose. An important point of recent debate has been the question of which regions of cellulose are most susceptible to glycosidic bond activation; as shown in Figure $\mathbf{6 A}$, internal mid-chain glycosidic linkages, a non-reducing end, and a levoglucosan end. Some studies have proposed that activation of glycosidic bonds occurs primarily at the nonreducing chain end, thus implying that cellulose unravels through a peeling mechanism - one chain end at a time. ${ }^{29}$ Others have instead proposed that internal mid-chain sites are active and undergo cleavage to form a shorter chain with a 


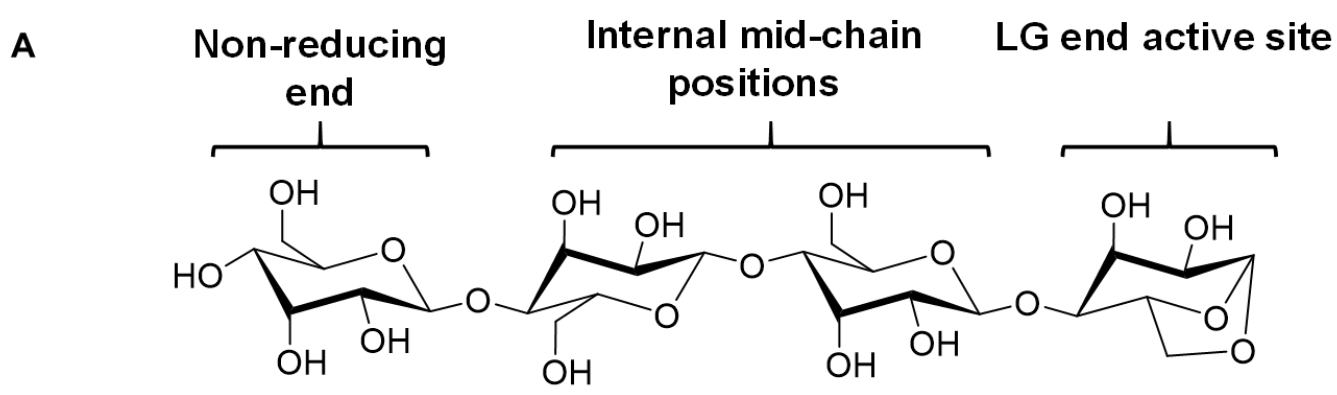

B

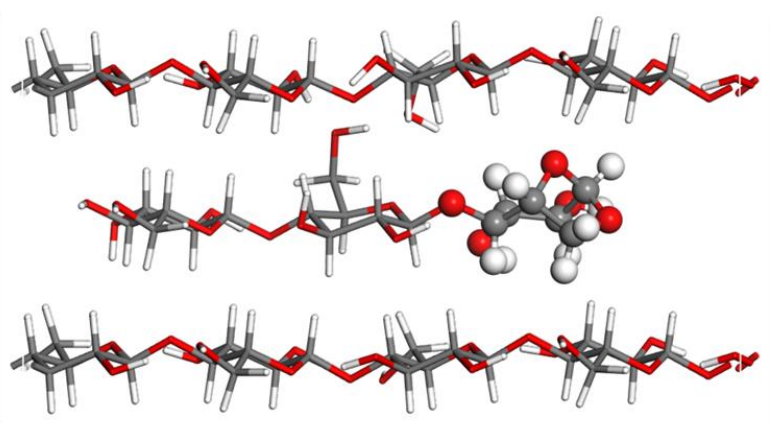

C

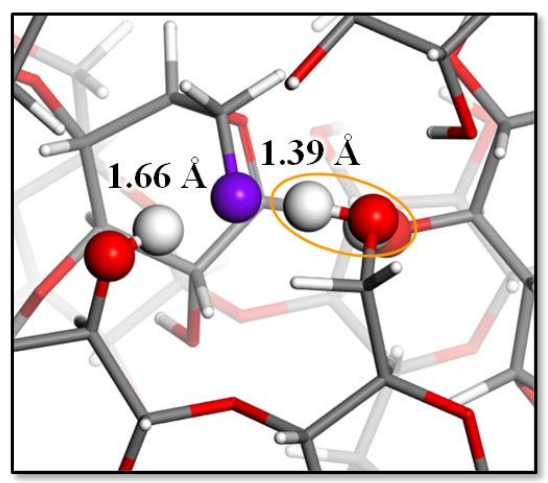

Figure 6. (A) Schematic of the 3 different sites where polymeric cleavage can occur (B) Model system of an active cellulosic chain with levoglucosan end group (shown in ball and stick form). (C) Magnified top view of transition state for hydroxyl-catalyzed activation at an internal mid-chain site at $t=15$ ps shows just two hydroxyl groups interacting with anionic $\mathrm{O}_{6}$ (purple sphere). Bridging hydroxyl circled red.

levoglucosan chain end. These levoglucosan chain end intermediates are proposed to be active, hence called 'active cellulose', and can subsequently undergo transglycosylation repeatedly to keep breaking glycosidic bonds and release levoglucosan. ${ }^{11,58}$ While the studies mentioned above have probed these two proposed routes of unravelling through density functional theory calculations, they have been carried out for the thermal, non-catalyzed transglycosylation activation pathway mentioned in section $\mathbf{1}$. The 3D confined environment that will likely exist around these three possible active sites - non-reducing chain end, internal mid-chain site, active cellulosic levoglucosan chain end was not considered. Since our model system already contains internal midchain sites, the hydroxyl-catalyzed activation was probed at these sites through constrained AIMD simulations in a manner similar to that used to examine the non-reducing chain end discussed in section 3.2. To probe the active cellulosic site, the structural model presented in Figure $\mathbf{3}$ was modified to change the chain-end glucose into a levoglucosan unit as shown in Figure $6 \mathbf{B}$.
The mean constraint force, $\left\langle\mathrm{F}_{\mathrm{c}}\right\rangle$, versus constrained coordinate plots similar to those shown in section 3.2 were obtained to evaluate the hydroxyl-catalyzed transglycosylation barriers at both the internal mid-chain sites and the active cellulosic sites (plots available in SI Figures S-4 and S-5). The thermodynamic integration of these forces over the constrained coordinates resulted in an intrinsic free energy barrier of $36.6 \mathrm{kcal} / \mathrm{mol}$ for reaction at the internal mid-chain site. This barrier is about $3.3 \mathrm{kcal} / \mathrm{mol}$ higher than the barrier reported for reaction at the non-reducing chain end. This suggests that the internal mid-chain sites are not as active as the non-reducing chain end. To rationalize this difference, we examined the structure for the transition state from the AIMD simulations for the internal mid-chain site. As shown in Figure $\mathbf{6 C}$, two hydroxyl groups interact with the anionic $\mathrm{O}_{6}$ center for the internal sites only, versus three hydroxyl groups that interacted with the non-reducing end. The fewer hydroxyl interactions could be the result of the slightly more confined environment at internal sites which prevent the reaction center from interacting with 


\section{Chem Rxiv ${ }^{\text {Th }}$}

three hydroxyl groups, as was observed in the nonreducing chain end case.

Table 1. Summary of free energy barriers and number of hydroxyl interactions at three active sites.

\begin{tabular}{|c|c|c|}
\hline $\begin{array}{c}\text { Location of Active } \\
\text { Site }\end{array}$ & $\begin{array}{c}\text { Intrinsic Free } \\
\text { Energy } \\
{\left[\mathrm{kcal} \mathrm{mol}^{-1}\right]}\end{array}$ & $\begin{array}{c}\text { Number of } \\
\text { H-bonding } \\
\text { Interactions }\end{array}$ \\
\hline $\begin{array}{l}\text { Non-reducing } \\
\text { chain end site }\end{array}$ & 33.3 & 3 \\
\hline $\begin{array}{l}\text { Internal mid-chain } \\
\text { site }\end{array}$ & 36.6 & 2 \\
\hline $\begin{array}{l}\text { Active cellulosic } \\
\text { site }\end{array}$ & 32.0 & 3 \\
\hline
\end{tabular}

The decrease in the number of $\mathrm{H}$-bonds from three to two in moving from the non-reducing end to the internal mid-chain site would help to explain the $\sim 3 \mathrm{kcal} / \mathrm{mol}$ increase in barriers as the strength of one $\mathrm{H}$-bond is typically around $4-5 \mathrm{kcal} / \mathrm{mol}$. Thermodynamic integration of constraint forces for the active cellulose site led to a free energy barrier of $32.0 \mathrm{kcal} / \mathrm{mol}$. The number of $\mathrm{H}$-bonding interactions for active cellulosic sites is three, which is similar to the number of interactions for the non-reducing chain end case and hence helps explain why it is more active than the mid-chain site. The slight difference in barriers between the non-reducing chain end site and the active cellulose site despite similar number of H-bonding interactions likely results from differences in monomer substitution - glucose (non-reducing chain end) versus levoglucosan (active cellulose site). These free energies and number of H-bonding interactions are summarized in Table 1. The AIMD results therefore suggest that non-reducing ends can be more active than internal mid-chain sites by virtue of being able to form stable $\mathrm{H}$-bonding networks with their surrounding hydroxyl groups. However, an active cellulosic site once formed, being more active relative to other sites, would likely continue to undergo subsequent activation faster than other sites and lead to cleaving of glycosidic bonds and release of levoglucosan.

4.0 Conclusions. Due to the importance of glycosidic bond activation in the pyrolytic reaction network, static density functional theory calculations as well as ab initio molecular dynamics simulations were conducted to evaluate the mechanisms for activating glycosidic bonds thermally as well as catalytically in the melt phase.
Transglycosylation was identified as a favorable mechanism in cellulose pyrolysis due to favorable interaction between an intramolecular hydroxymethyl group and the lone pair electrons on the glycosidic oxygen. This insight led to a new mechanism for activating glycosidic bonds with inverted stereochemistry ( $\beta$ versus $\alpha)$ which involved the formation of an epoxide intermediate. Through static DFT calculations on a simple model compound (maltose), a different intramolecular hydroxyl group $\left(\mathrm{C}_{2}\right.$ hydroxyl) was shown to carry out a similar nucleophilic attack on the $\alpha$ glycosidic bond as is done by the hydroxymethyl $\left(\mathrm{O}_{6} \mathrm{H}\right)$ group on the $\beta$ glycosidic bond, thus leading to a similar activation energy $(52.4 \mathrm{kcal} / \mathrm{mol})$. Free energies calculated through the quasi-harmonic oscillator approach also reveal an entropic gain of 6.7 $\mathrm{cal} / \mathrm{mol}-\mathrm{K}$ which is comparable with entropic gains typically observed in thermal cracking. Building on these thermal pathways, we considered pathways that are catalyzed in the condensed phase by local hydroxyl groups present in the reaction environment which were shown to influence the reaction dynamics of activating glycosidic bonds in cellulose. With the use of constrained ab initio molecular dynamics (AIMD), up to three vicinal hydroxyl groups were shown to dynamically arrange themselves to form nested pockets that can stabilize the transition state significantly over the reactant state through hydrogen bonding networks and electrostatic interactions. While these environments lead to significant electronic stabilization, they were also shown to lead to a small entropic penalty. The number of these interactions and the ability to form such networks directly correlated with activity of different regions of cellulose. The intrinsic free energy barriers calculated for the three possible active sites that were probed - non-reducing chain end (33.3 $\mathrm{kcal} / \mathrm{mol})$, internal mid-chain site $(36.6 \mathrm{kcal} / \mathrm{mol})$ and active cellulose site $(32.0 \mathrm{kcal} / \mathrm{mol})$ - revealed that the order for activity of the different regions are active cellulosic sites $\sim$ non-reducing chain ends > internal mid-chain sites. In summary, the results provide further insights into intra-molecular and intermolecular factors that influence glycosidic activation energetics, reaction dynamics and ultimately the reaction kinetics of cellulose deploymerization. 
Acknowledgements. We acknowledge support from the U.S. Department of Energy, Office of Basic Energy Science Catalysis (DE-SC0016346).

Keywords. Cellulose, Transglycosylation, Glycosidic Bond, Hydroxyl, DFT, AIMD

Supporting Information. The supporting information contains additional structures of intermediates and transition states that have not been displayed in the main text. Plots of constraint force vs reaction coordinate are also provided for longer times as well as the other sites that have been explored in section 3.3 of the manuscript.

\section{References.}

(1) Negahdar, L.; Gonzalez-Quiroga, A.; Otyuskaya, D.; Toraman, H. E.; Liu, L.; Jastrzebski, J. T. B. H.; Van Geem, K. M.; Marin, G. B.; Thybaut, J. W.; Weckhuysen, B. M. Characterization and Comparison of Fast Pyrolysis Bio-Oils from Pinewood, Rapeseed Cake, and Wheat Straw Using13C NMR and Comprehensive GC $\times$ GC. ACS Sustain. Chem. Eng. 2016, 4 (9), 4974-4985.

(2) Black, B. A.; Michener, W. E.; Ramirez, K. J.; Biddy, M. J.; Knott, B. C.; Jarvis, M. W.; Olstad, J.; Mante, O. D.; Dayton, D. C.; Beckham, G. T. Aqueous Stream Characterization from Biomass Fast Pyrolysis and Catalytic Fast Pyrolysis. ACS Sustain. Chem. Eng. 2016, 4 (12), 6815-6827.

(3) Stamm, A. J. Thermal Degradation of Wood and Cellulose. Ind. Eng. Chem. 1956, 48 (3), 413417.

(4) Bridgwater, A. V. Renewable Fuels and Chemicals by Thermal Processing of Biomass. Chem. Eng. J. 2003, 91 (2-3), 87-102.

(5) Naik, S. N.; Goud, V. V.; Rout, P. K.; Dalai, A. K. Production of First and Second Generation Biofuels: A Comprehensive Review. Renew. Sustain. Energy Rev. 2010, 14 (2), 578-597.

(6) Mohan, D.; Pittman, C. U.; Steele, P. H. Pyrolysis of Wood/Biomass for Bio-Oil: A Critical Review. Energy and Fuels 2006, 20 (3), 848-889.

(7) Chundawat, S. P. S.; Beckham, G. T.; Himmel, M. E.; Dale, B. E. Deconstruction of Lignocellulosic Biomass to Fuels and Chemicals. Annu. Rev. Chem. Biomol. Eng. 2011, 2 (1), 121145.

(8) Álvarez, C.; Reyes-Sosa, F. M.; Díez, B.
Enzymatic Hydrolysis of Biomass from Wood. Microb. Biotechnol. 2016, 9 (2), 149-156.

(9) Biomass Pyrolysis to Hydrocarbon Fuels in the Petroleum Refining Context: Cooperative Research and Development Final Report, CRADA Number CRD-12-500,.

(10) Mettler, M. S.; Vlachos, D. G.; Dauenhauer, P. J. Top Ten Fundamental Challenges of Biomass Pyrolysis for Biofuels. Energy Environ. Sci. 2012, 5 (7), 7797.

(11) Vinu, R.; Broadbelt, L. J. A Mechanistic Model of Fast Pyrolysis of Glucose-Based Carbohydrates to Predict Bio-Oil Composition. Energy Environ. Sci. 2012, 5 (12), 9808.

(12) Assary, R. S.; Curtiss, L. A. Thermochemistry and Reaction Barriers for the Formation of Levoglucosenone from Cellobiose. ChemCatChem 2012, 4 (2), 200-205.

(13) Mayes, H. B.; Broadbelt, L. J. Unraveling the Reactions That Unravel Cellulose. J. Phys. Chem. A 2012, 116 (26), 7098-7106.

(14) Seshadri, V.; Westmoreland, P. R. Concerted Reactions and Mechanism of Glucose Pyrolysis and Implications for Cellulose Kinetics. J. Phys. Chem. A 2012, 116 (49), 11997-12013.

(15) Hosoya, T.; Nakao, Y.; Sato, H.; Kawamoto, H.; Sakaki, S. Thermal Degradation of Methyl $\beta$ D-Glucoside. A Theoretical Study of Plausible Reaction Mechanisms. J. Org. Chem. 2009, 74 (17), 6891-6894.

(16) Hosoya, T.; Sakaki, S. Levoglucosan Formation from Crystalline Cellulose: Importance of a Hydrogen Bonding Network in the Reaction. ChemSusChem 2013, 6 (12), 23562368.

(17) Ponder, G. R.; Richards, G. N. A Review of Some Recent Studies on Mechanisms of Pyrolysis of Polysaccharides. Biomass and Bioenergy 1994, 7 (1-6), 1-24.

(18) Lowary, T. L.; Richards, G. N. Mechanisms of Pyrolysis of Polysaccharides: Cellobiitol as a Model for Cellulose. Carbohydr. Res. 1990, 198 (1), 79-89.

(19) Shen, D. K.; Gu, S. The Mechanism for Thermal Decomposition of Cellulose and Its Main Products. Bioresour. Technol. 2009, 100 (24), 6496-6504.

(20) Patwardhan, P. R. Understanding the Product Distribution from Biomass Fast Pyrolysis. Chem. Eng. 2010, Ph. D. (Iowa State).

(21) Mettler, M. S.; Mushrif, S. H.; Paulsen, A. D.; 


\section{Chem Rxiv ${ }^{\text {Th }}$}

Javadekar, A. D.; Vlachos, D. G.; Dauenhauer, P. J. Revealing Pyrolysis Chemistry for Biofuels Production: Conversion of Cellulose to Furans and Small Oxygenates. Energy Environ. Sci. 2012, 5 (1), 5414-5424.

(22) Mettler, M. S.; Paulsen, A. D.; Vlachos, D. G.; Dauenhauer, P. J. The Chain Length Effect in Pyrolysis: Bridging the Gap between Glucose and Cellulose. Green Chem. 2012, 14 (5), 1284.

(23) Mayes, H. B.; Nolte, M. W.; Beckham, G. T.; Shanks, B. H.; Broadbelt, L. J. The Alpha-Bet(a) of Glucose Pyrolysis: Computational and Experimental Investigations of 5Hydroxymethylfurfural and Levoglucosan Formation Reveal Implications for Cellulose Pyrolysis. ACS Sustain. Chem. Eng. 2014, 2 (6), 1461-1473.

(24) Maduskar, S.; Maliekkal, V.; Neurock, M.; Dauenhauer, P. J. On the Yield of Levoglucosan from Cellulose Pyrolysis. ACS Sustain. Chem. Eng. 2018, 6 (5), 7017-7025.

(25) Lindstrom, J. K.; Proano-aviles, J.; Peterson, C. A.; Stansell, J. S.; Johnston, P. A.; Brown, R. C. Competing Reactions Limit Levoglucosan Yield during Fast Pyrolysis of Cellulose. Green Chem. 2018, 21, 178-186.

(26) Yu, Z. J.; Easton, M. W.; Murria, P.; Xu, L.; Ding, D.; Jiang, Y.; Zhang, J.; Kenttämaa, H. I. Molecular-Level Understanding of the Major Fragmentation Mechanisms of Cellulose Fast Pyrolysis: An Experimental Approach Based on Isotopically Labeled Model Compounds. J. Org. Chem. 2019, 84 (11), 7037-7050.

(27) Seshadri, V.; Westmoreland, P. R. Roles of Hydroxyls in the Noncatalytic and Catalyzed Formation of Levoglucosan from Glucose. Catal. Today 2016, 269, 110-121.

(28) Paulsen, A. D.; Mettler, M. S.; Dauenhauer, P. J. The Role of Sample Dimension and Temperature in Cellulose Pyrolysis. Energy and Fuels 2013, 27 (4), 2126-2134.

(29) Krumm, C.; Pfaendtner, J.; Dauenhauer, P. J. Millisecond Pulsed Films Unify the Mechanisms of Cellulose Fragmentation. Chem. Mater. 2016, 28 (9), 3108-3114.

(30) Zhu, C.; Krumm, C.; Facas, G. G.; Neurock, M.; Dauenhauer, P. J. Energetics of Cellulose and Cyclodextrin Glycosidic Bond Cleavage. React. Chem. Eng. 2017, 2 (2), 201-214.

(31) Maliekkal, V.; Maduskar, S.; Saxon, D. J.; Nasiri, M.; Reineke, T. M.; Neurock, M.;
Dauenhauer, P. Activation of Cellulose via Cooperative Hydroxyl-Catalyzed Transglycosylation of Glycosidic Bonds. ACS Catal. 2018, 9, 1943-1955.

(32) Westmoreland, P. R. Pyrolysis Kinetics for Lignocellulosic Biomass-to-Oil from Molecular Modeling. Curr. Opin. Chem. Eng. 2019, 23, 123-129.

(33) Kresse, G.; Hafner, J. Ab Initio Molecular Dynamics for Liquid Metals. Phys. Rev. B 1993, 47 (1), 558-561.

(34) Kresse, G.; Hafner, J. Ab Initio MolecularDynamics Simulation of the Liquid-MetalAmorphous-Semiconductor Transition in Germanium. Phys. Rev. B 1994, 49 (20), 1425114269.

(35) Kresse, G.; Furthmüller, J. Efficient Iterative Schemes for Ab Initio Total-Energy Calculations Using a Plane-Wave Basis Set. Phys. Rev. B 1996, 54 (16), 11169-11186.

(36) Perdew, J. P.; Burke, K.; Ernzerhof, M. Generalized Gradient Approximation Made Simple. Phys. Rev. Lett. 1996, 77 (18), 38653868.

(37) Grimme, S. Semiempirical GGA-Type Density Functional Constructed with a LongRange Dispersion Correction. J Comput Chem 2006, 27, 1787-1799.

(38) Grimme, S. Density Functional Theory with London Dispersion Corrections. Wiley Interdiscip. Rev. Comput. Mol. Sci. 2011, 1 (2), 211-228.

(39) Kresse, G.; Joubert, D. From Ultrasoft Pseudopotentials to the Projector AugmentedWave Method. Phys. Rev. B 1999, 59 (3), 17581775.

(40) Blöchl, P. E. Projector Augmented-Wave Method. Phys. Rev. B 1994, 50 (24), 1795317979.

(41) Pack, J. D.; Monkhorst, H. J. "special Points for Brillouin-Zone Integrations"-a Reply. Phys. Rev. B 1977, 16 (4), 1748-1749.

(42) Henkelman, G.; Jónsson, H. Improved Tangent Estimate in the Nudged Elastic Band Method for Finding Minimum Energy Paths and Saddle Points Improved Tangent Estimate in the Nudged Elastic Band Method for Finding Minimum Energy Paths and Saddle Points. J. Chem. Phys. 2000, 9978 (113), 9978-9985.

(43) Henkelman, G.; Uberuaga, B. P.; Jónsson, H.; Henkelman, G. A Climbing Image Nudged 
Elastic Band Method for Finding Saddle Points and Minimum Energy Paths A Climbing Image Nudged Elastic Band Method for Finding Saddle Points and Minimum Energy Paths. J. Chem. Phys. 2011, 113 (2000), 9901-9905.

(44) Henkelman, G.; Jónsson, H. A Dimer Method for Finding Saddle Points on High Dimensional Potential Surfaces Using Only First Derivatives. J. Chem. Phys. 1999, 111, 7010-7022.

(45) Ribeiro, R. F.; Marenich, A. V; Cramer, C. J.; Truhlar, D. G. Use of Solution-Phase Vibrational Frequencies in Continuum Models for the Free Energy of Solvation. 2011, 14556-14562.

(46) Nishiyama, Y.; Langan, P.; Chanzy, H. Crystal Structure and Hydrogen-Bonding System in Cellulose I $\beta$ from Synchrotron X-Ray and Neutron Fiber Diffraction. J. Am. Chem. Soc. 2002, 124 (31), 9074-9082.

(47) Langan, P.; Sukumar, N.; Nishiyama, Y.; Chanzy, H. Synchrotron X-Ray Structures of Cellulose I $\beta$ and Regenerated Cellulose II at Ambient Temperature and $100 \mathrm{~K}$. Cellulose 2005, 12 (6), 551-562.

(48) Hoover, W. G. Canonical Dynamics: Equilibrium Phase-Space Distributions. Phys. Rev. A 1985, 31 (3), 1695-1697.

(49) Nose, S. A Unified Formulation of the Constant Temperature Molecular Dynamics Methods. J. Chem. Phys 1984, 81 (1), 511-519.

(50) $\mathrm{Bu}^{2}, \mathrm{~T}$. Proton Exchange of Small Hydrocarbons over Acidic Chabazite: Ab Initio Study of Entropic Effects. 2007, 250, 171-183.

(51) Ryckaert, J. P.; Ciccotti, G.; Berendsen, H. J. C. Numerical Integration of the Cartesian Equations of Motion of a System with Constraints: Molecular Dynamics of n-Alkanes. J. Comput. Phys. 1977, 23 (3), 327-341.

(52) Coluzza, I.; Sprik, M.; Ciccotti, G. Constrained Reaction Coordinate Dynamics for Systems with Constraints. Mol. Phys. 2003, 101 (18), 28852894.

(53) Sprik, M.; Ciccotti, G. Free Energy from Constrained Molecular Dynamics. J. Chem. Phys. 1998, 109 (18), 7737-7744.

(54) Ciccotti, G.; Kapral, R.; Vanden-eijnden, E. Blue Moon Sampling, Vectorial Reaction Coordinates , and Unbiased Constrained Dynamics. ChemPhysChem 2005, 6, 1809-1814. (55) Otter, W. K. Den; Briels, W. J. The Calculation of Free-Energy Differences by Constrained Molecular-Dynamics Simulations The
Calculation of Free-Energy Differences by Constrained Molecular-Dynamics Simulations. J. Chem. Phys. 1998, 109 (11), 4139-4146.

(56) Mellmer, M. A.; Sanpitakseree, C.; Demir, B.; Bai, P.; Ma, K.; Neurock, M.; Dumesic, J. A. Solvent-Enabled Control of Reactivity for Liquid-Phase Reactions of Biomass-Derived Compounds. Nat. Catal. 2018, 1 (3), 199-207.

(57) Mellmer, M. A.; Sanpitakseree, C.; Demir, B.; Ma, K.; Elliott, W. A.; Bai, P.; Johnson, R. L.; Walker, T. W.; Shanks, B. H.; Rioux, R. M.; et al. Effects of Chloride Ions in Acid-Catalyzed Biomass Dehydration Reactions in Polar Aprotic Solvents. Nat. Commun. 2019, 10 (1132), 1-10.

(58) Agarwal, V.; Dauenhauer, P. J.; Huber, G. W.; Auerbach, S. M. Ab Initio Dynamics of Cellulose Pyrolysis: Nascent Decomposition Pathways at 327 and 600 C. J. Am. Chem. Soc. 2012, 134 (36), 14958-14972. 


\title{
SUPPORTING INFORMATION
}

\section{Glycosidic C-O Bond Activation in Cellulose Pyrolysis: Alpha Versus Beta and Condensed Phase Hydroxyl-Catalytic Scission}

\author{
Vineet Maliekkal ${ }^{1}$, Paul Dauenhauer ${ }^{1}$, Matthew Neurock ${ }^{1 *}$ \\ ${ }^{1}$ University of Minnesota, Department of Chemical Engineering, Amundson Hall, 425 Washington Ave \\ SE, Minneapolis, MN 55455, USA. \\ *Corresponding Author: mneurock@umn.edu
}

Number of Pages: 7

Number of Figures: 6

S1. Additional structures for $\alpha$ glycosidic bond activation mechanism

S2. Transition state structure for epoxide to levoglucosan conversion without assistance

S3. Mean constraint force vs reaction coordinate plots at longer times

S4. Additional mean constraint force vs reaction coordinate plots for other sites

S5. Snapshots of transition state at active cellulose site 


\section{S1. Additional structures for $\alpha$ glycosidic bond activation mechanism}

A

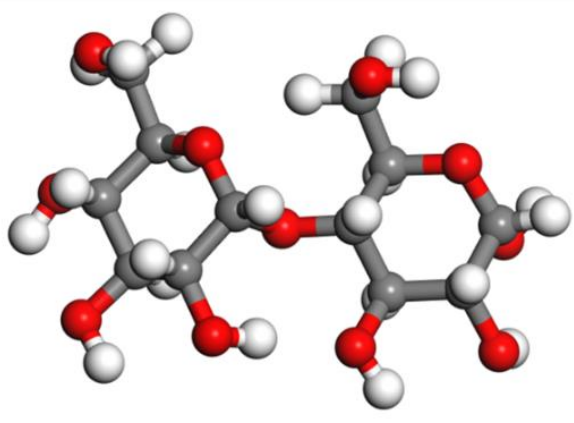

C

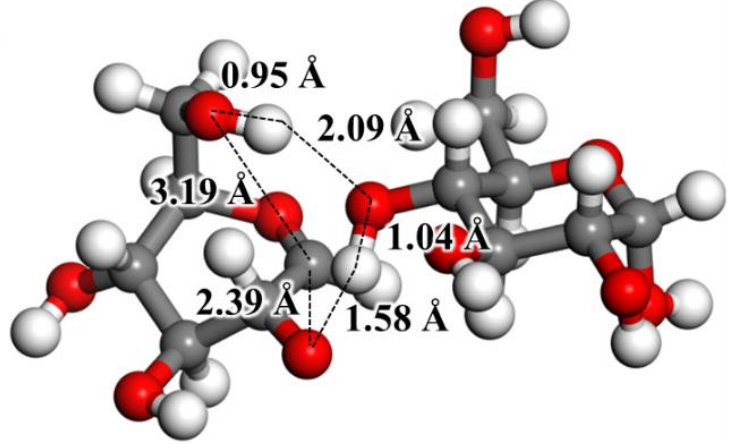

B
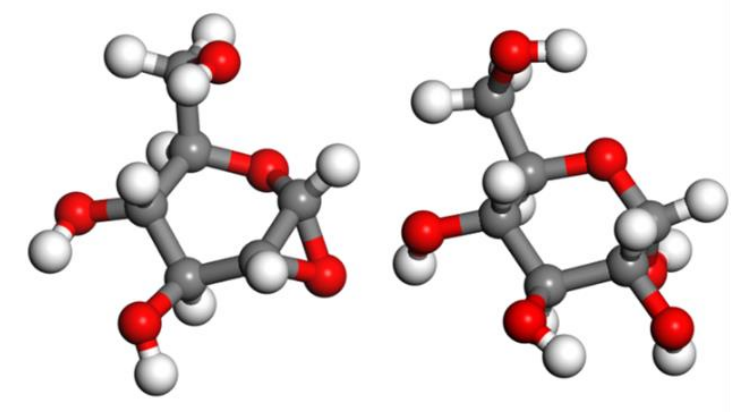

D

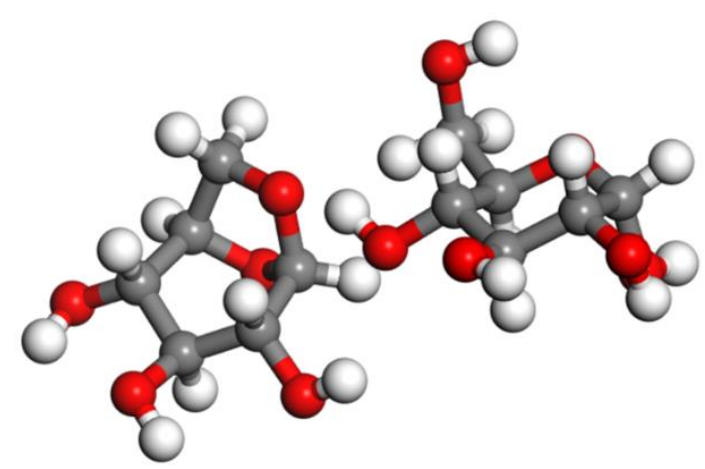

Figure S-1. Optimized structures for (A) maltose reactant (B) epoxide + glucose intermediate (C) transition state for conversion of epoxide to levoglucosan complex (D) levoglucosan + glucose product complex

Note that optimized transition state for first step of this mechanism is already shown in main text in Figure 2B. By comparing Figures S1B, S1C and S1D we see that the transition state for second step of this mechanism is an early transition state. This is evident by the bond lengths in the transition state which reveal that proton transfer and bicyclic ring formation have yet to take place. 


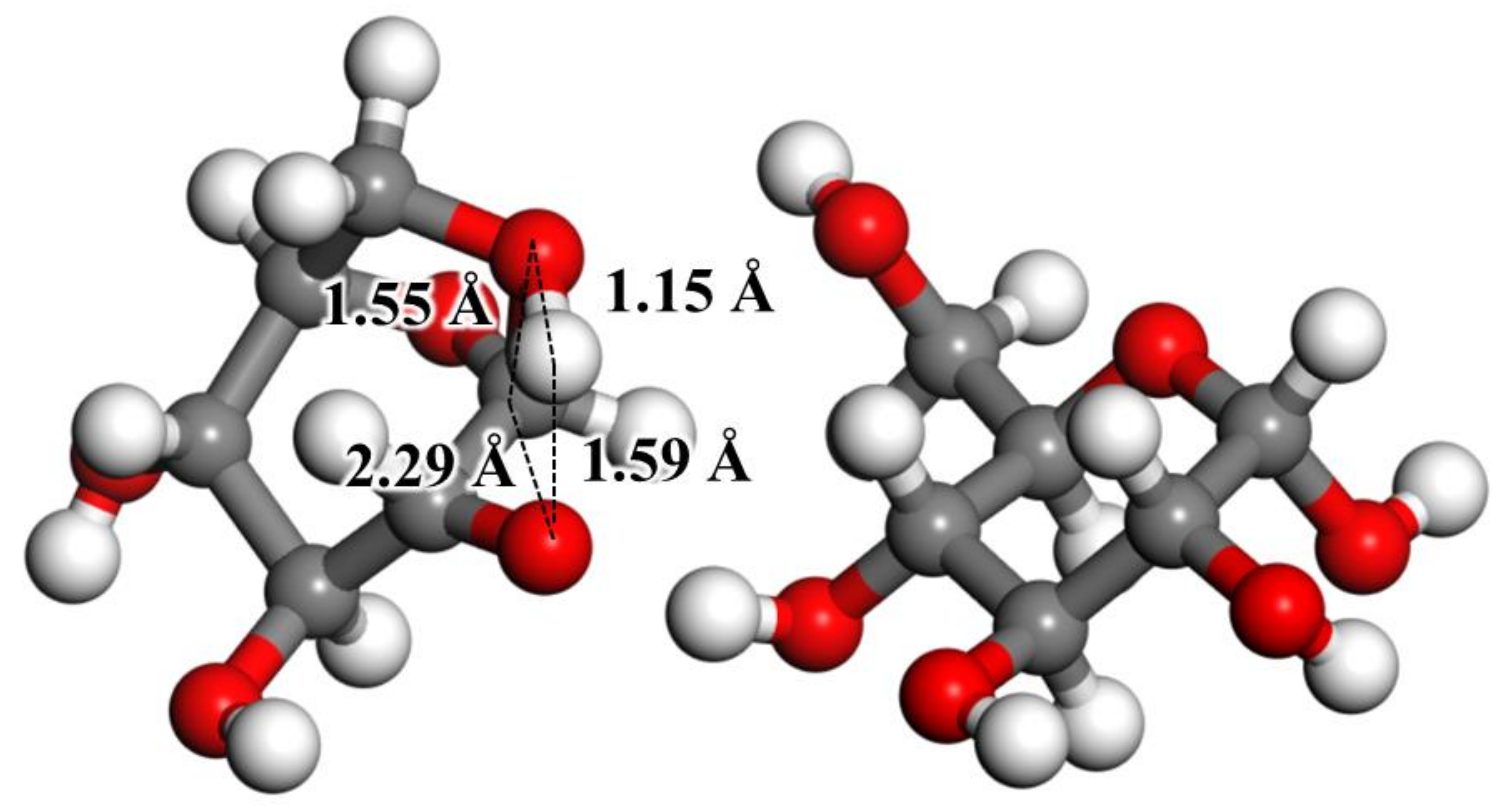

Figure S-2. Optimized structures of transition state for converting the epoxide intermediate to the levoglucosan intermediate without assistance from detached glucose unit

The optimized structure for this transition state is highly extremely strained thus leading to the higher barrier for this step. This indicates that a hydroxyl group from detached glucose unit may be necessary to carry out the second step of converting epoxide to levoglucosan. 


\section{S3. Mean constraint force vs reaction coordinate plots at longer times}
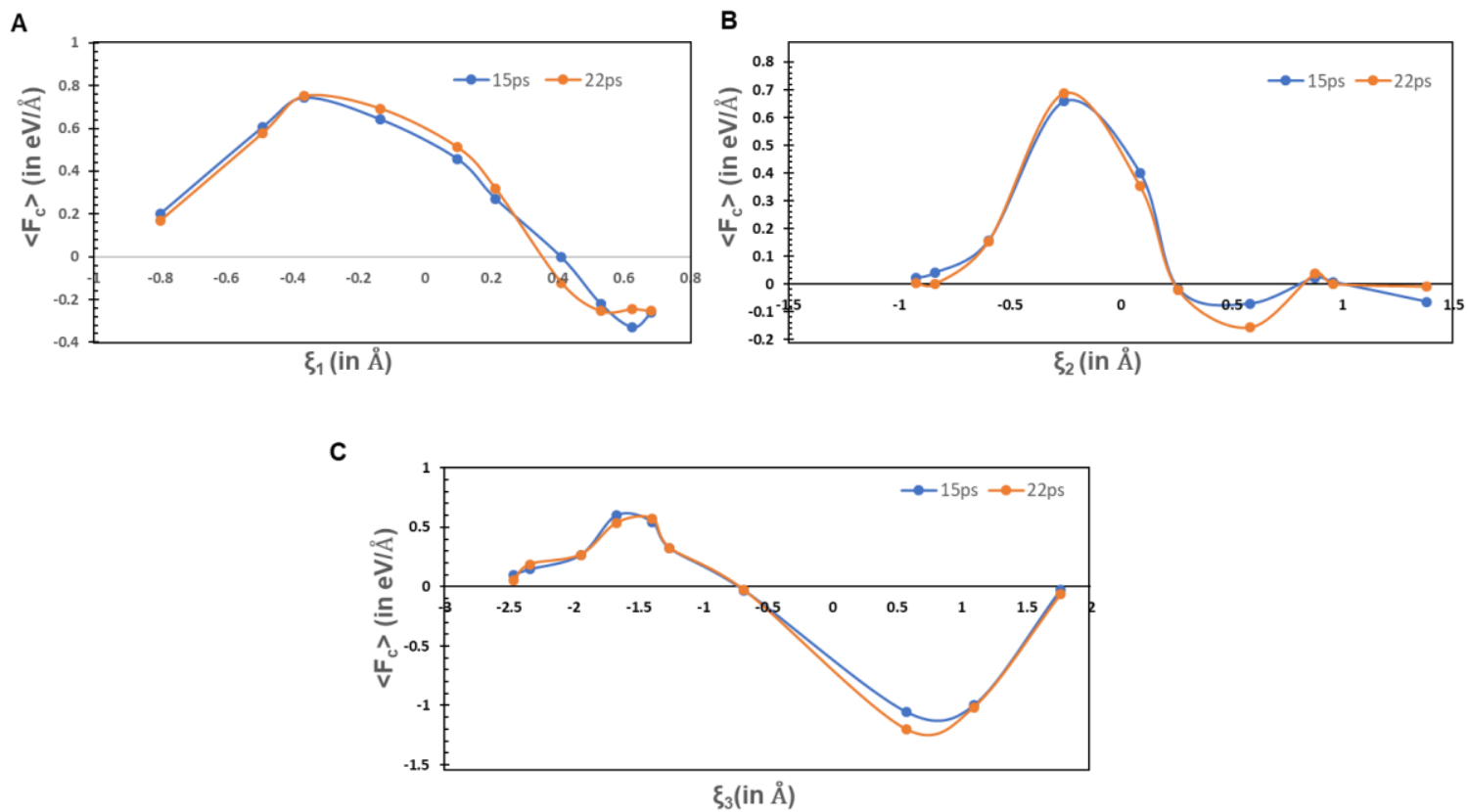

Figure S-3. Mean constraint force vs. reaction coordinate plots for (A) $\xi_{1}$ (B) $\xi_{2}$ and (C) $\xi_{3}$ obtained by running trajectories for $15 \mathrm{ps}$ (blue) and $22 \mathrm{ps}$ (red). Similarity of the Force - coordinates curves at for both sets of trajectories indicates that $15 \mathrm{ps}$ is sufficient time for sampling of data. 


\section{S4. Additional mean constraint force vs reaction coordinate plots for other sites}

Presented below are the constraint force vs. coordinate curves for the internal mid-chain sites as well as the LG end active cellulosic sites. As we are interested in the free energy of activation, we sample reaction coordinates until the force curve is close to or crosses zero after attaining a maxima thus indicating a transition state.

A

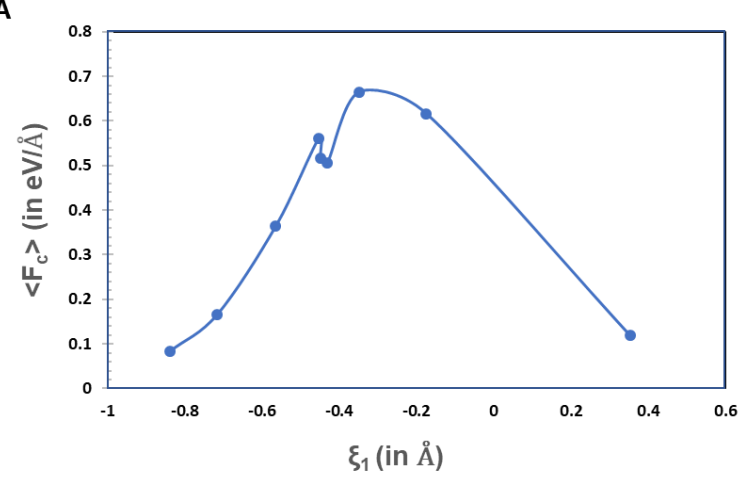

B

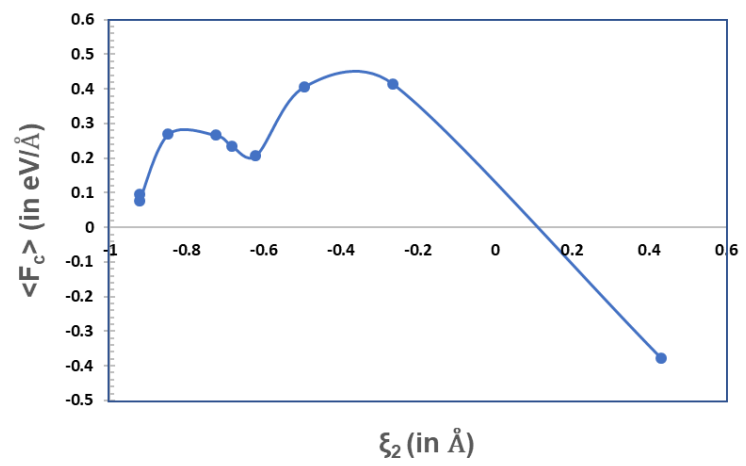

C

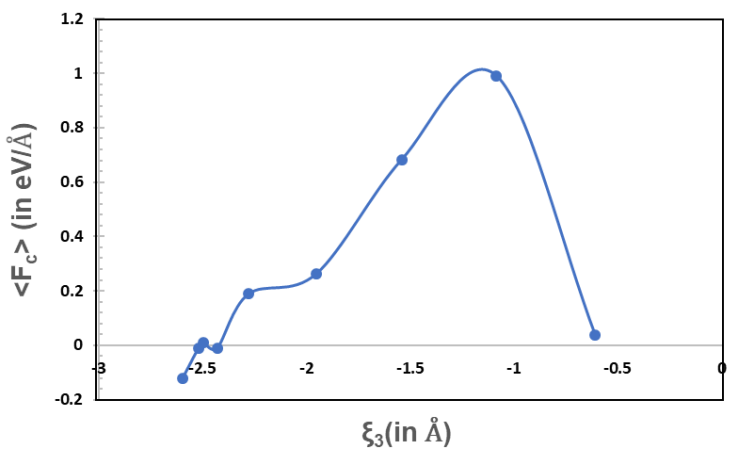

Figure S-4. Mean constraint force vs reaction coordinate plots for (A) $\xi_{1}(\mathbf{B}) \xi_{2}$ and (C) $\xi_{3}$ at the midchain internal site. Points beyond the transition state are not considered. 
A

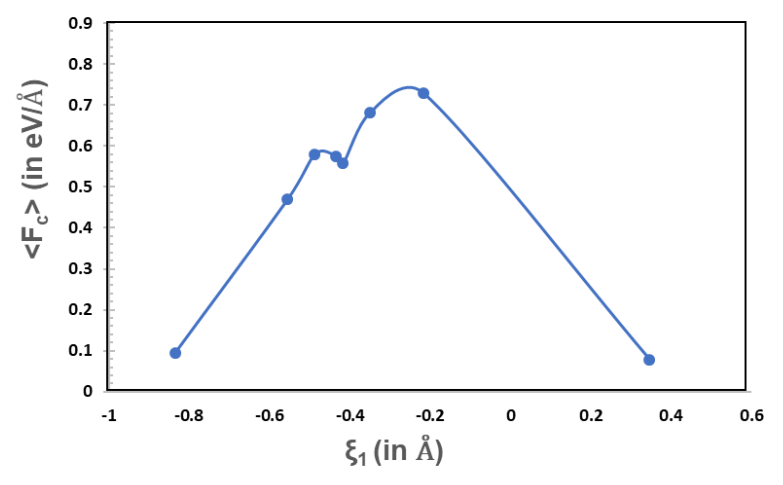

B

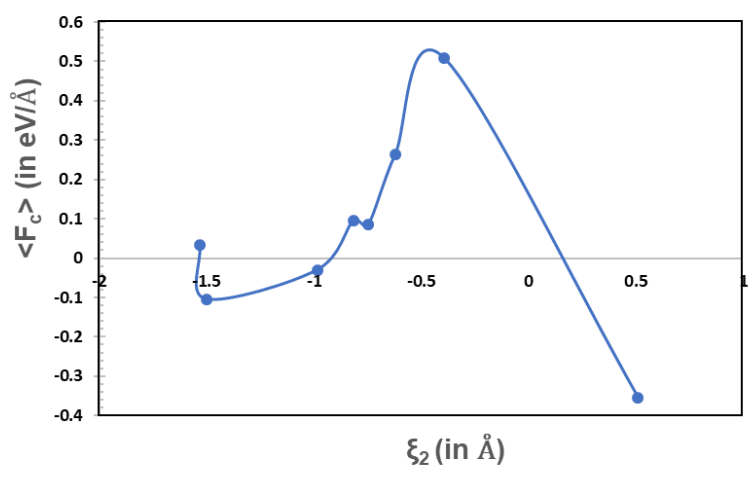

C

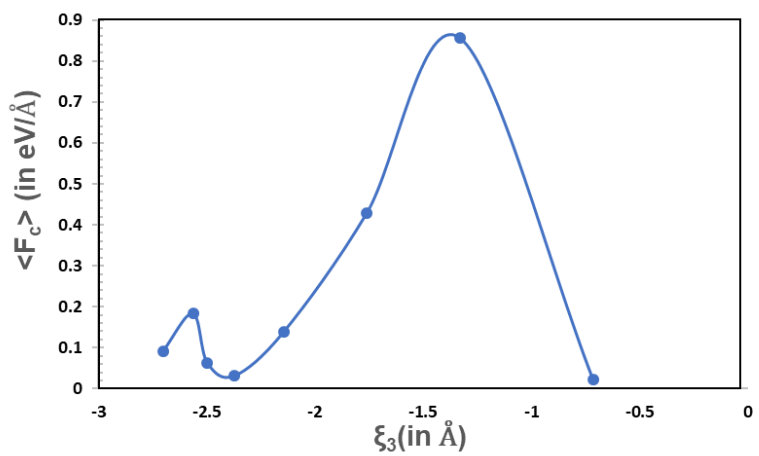

Figure S-5. Mean constraint force vs reaction coordinate plots for (A) $\xi_{1}$ (B) $\xi_{2}$ and (C) $\xi_{3}$ at the LG end active cellulose sites. Points beyond the transition state are not considered. 
S5. Snapshots of transition state at an active cellulose site

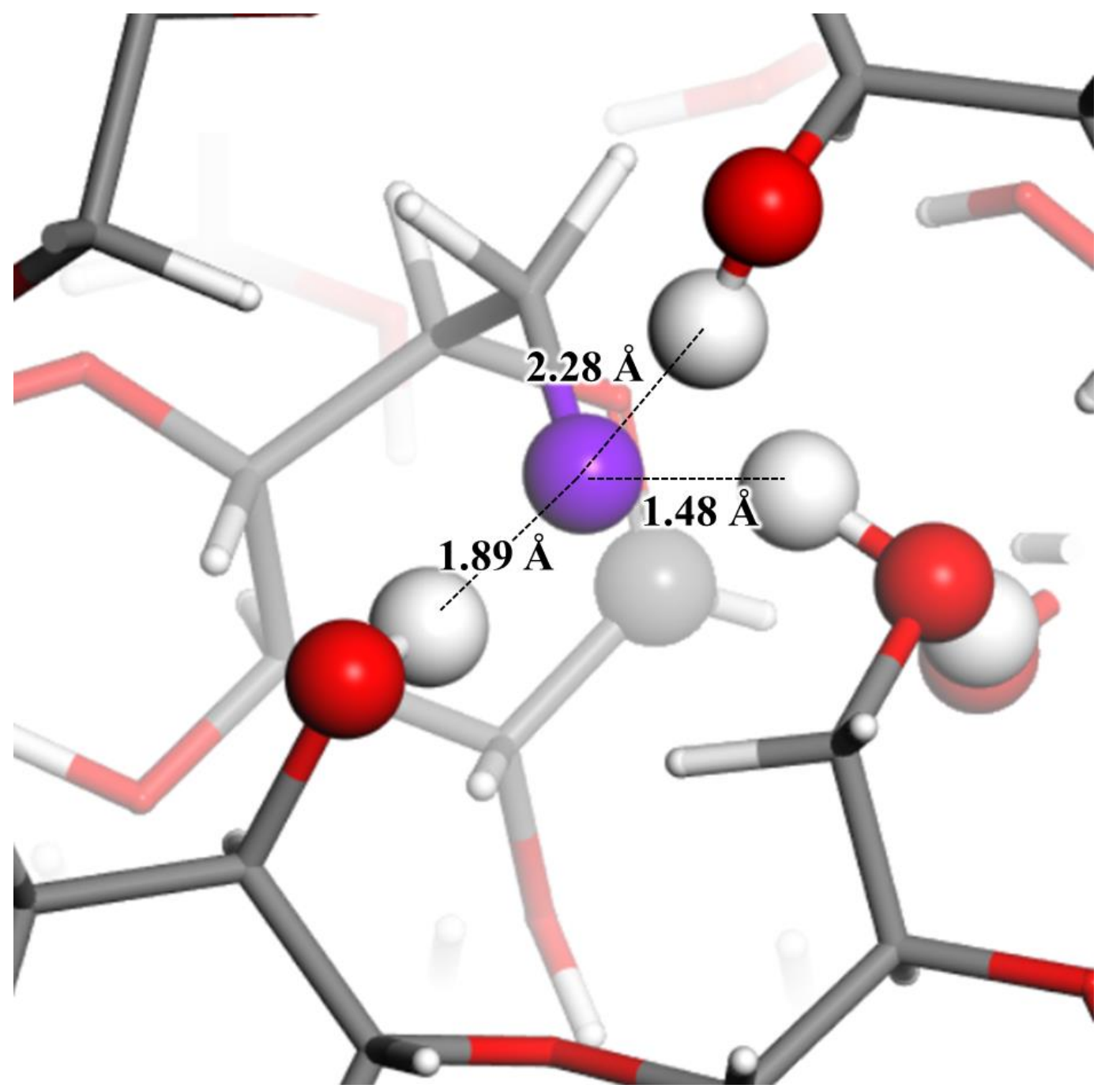

Figure S-6. Snapshot of transition state along the MD trajectory reveals that $3 \mathrm{H}$-bonds interact with the negatively charged $\mathrm{O}_{6}$ center for the $\mathrm{LG}$ end active cellulose site 
